WSU-HEP-0404

MCTP-04-36

hep-ph/0407004

\title{
Spectator effects and lifetimes of heavy hadrons
}

\author{
Fabrizio Gabbiani ${ }^{1}$, Andrei I. Onishchenko ${ }^{1}$ and Alexey A. Petrov ${ }^{1,2}$ \\ ${ }^{1}$ Department of Physics and Astronomy, Wayne State University, \\ Detroit, MI 48201 \\ ${ }^{2}$ Michigan Center for Theoretical Physics, University of Michigan, \\ Ann Arbor, MI 48109
}

\begin{abstract}
We present a calculation of subleading $1 / m_{b}$ corrections to spectator effects in the ratios of beauty hadron lifetimes in heavy-quark expansion. We find that these effects are sizable and should be taken into account in systematic analyses of heavy hadron lifetimes. In particular, the inclusion of $1 / m_{b}$ corrections brings into agreement the theoretical predictions and experimental observations of the ratio of lifetimes of $\Lambda_{b}$-baryon and $B_{d}$ meson. We obtain $\tau\left(B_{u}\right) / \tau\left(B_{d}\right)=1.06 \pm 0.02, \tau\left(B_{s}\right) / \tau\left(B_{d}\right)=1.00 \pm 0.01, \tau\left(\Lambda_{b}\right) / \tau\left(B_{d}\right)=0.86 \pm 0.05$.
\end{abstract}




\section{Introduction}

The hierarchy of lifetimes of heavy hadrons can be understood in the heavy-quark expansion (HQE), which makes use of the disparity of scales present in the decays of hadrons containing bquarks. HQE predicts the ratios of lifetimes of beauty mesons, which agree with the experimental observations well within experimental and theoretical uncertainties. Most recent analyses give $[1,2]$

$$
\begin{array}{ll}
\tau\left(B_{u}\right) /\left.\tau\left(B_{d}\right)\right|_{e x}=1.085 \pm 0.017, & \tau\left(B_{u}\right) /\left.\tau\left(B_{d}\right)\right|_{t h}=1.07 \pm 0.03 \\
\tau\left(B_{s}\right) /\left.\tau\left(B_{d}\right)\right|_{e x}=0.951 \pm 0.038, & \tau\left(B_{s}\right) /\left.\tau\left(B_{d}\right)\right|_{t h}=1.00 \pm 0.02
\end{array}
$$

which show evidence of excellent agreement of theoretical predictions and experimental measurements. This agreement also provides us with some confidence that quark-hadron duality, which states that smeared partonic amplitudes can be replaced by the hadronic ones, is expected to hold in inclusive decays of heavy flavors. The heavy-quark expansion also provides a qualitative understanding of the hierarchy of lifetimes of charmed hadrons. Since the expansion parameter there is much larger, only qualitative agreement is expected.

The only remaining problem appears in the ratios of lifetimes of heavy mesons and baryons. For instance, the low experimental value of the ratio $\tau\left(\Lambda_{b}\right) / \tau\left(B_{d}\right)$ has long been a puzzle for the theory. The latest compilation of experimental observations [2] suggests that

$$
\tau\left(\Lambda_{b}\right) /\left.\tau\left(B_{d}\right)\right|_{e x}=0.797 \pm 0.053
$$

which is inconsistent with the first theoretical predictions of this ratio, $0.90-0.95[3,4,5]$ at the level of approximately $2 \sigma$. Only recent next-to-leading order (NLO) calculations of perturbative QCD [6] and $1 / m_{b}$ corrections [7] to spectator effects significantly reduced the discrepancy, yielding, as reported in [9],

$$
\tau\left(\Lambda_{b}\right) /\left.\tau\left(B_{d}\right)\right|_{t h}=0.88 \pm 0.05
$$

which appears in some agreement with experimental data, mainly due to sizable theoretical and experimental uncertainties. The problem of $\tau\left(\Lambda_{b}\right) / \tau\left(B_{d}\right)$ ratio could reappear again if future measurements at Fermilab and CERN would find the mean value to stay the same with error bars shrinking. Upcoming Fermilab measurements of $\Lambda_{b}$ lifetime could shed more light on the experimental side of this issue.

Here we expand our calculation of subleading contributions to spectator effects in the $1 / m_{b}$ expansion [7] to study their impact on the ratios of lifetimes of heavy mesons. We include the full charm quark-mass dependence, generalizing our result of Ref. [7]. We also discuss the convergence of the $1 / m_{b}$ expansion in the analysis of spectator effects by estimating next, $1 / m_{b}^{2}$-suppressed corrections to the spectator effects in factorization.

This paper is organized as follows. We set up the relevant formalism and argue for the need to compute subleading $1 / m_{b}$ corrections to spectator effects in Sect. 2 . In Sect. 3 we discuss the impact of $1 / m_{b}$ corrections to lifetime ratios of heavy mesons and baryons and assess the convergence of the $1 / m_{b}$ expansion. We present the complete results from $1 / m_{b}^{2}$ corrections to spectator effects. Finally, we present our conclusions in Sect. 4. Some of the relevant formulas are shown in the Appendices. 


\section{Formalism}

The inclusive decay rate of a heavy hadron $H_{b}$ is most conveniently computed by employing the optical theorem to relate the decay width to the imaginary part of the forward matrix element of the transition operator:

$$
\Gamma\left(H_{b}\right)=\frac{1}{2 M_{H_{b}}}\left\langle H_{b}|\mathcal{T}| H_{b}\right\rangle, \quad \mathcal{T}=\operatorname{Im} i \int d^{4} x T\left\{H_{\mathrm{eff}}(x) H_{\mathrm{eff}}(0)\right\} .
$$

Here $H_{\text {eff }}$ represents an effective $\Delta B=1$ Hamiltonian,

$$
H_{\mathrm{eff}}=\frac{4 G_{F}}{\sqrt{2}} V_{c b} \sum_{d^{\prime}=d, s, u^{\prime}=u, c} V_{u^{\prime} d^{\prime}}^{*}\left[C_{1}(\mu) Q_{1}^{u^{\prime} d^{\prime}}(\mu)+C_{2}(\mu) Q_{2}^{u^{\prime} d^{\prime}}(\mu)\right]+\text { h.c. }
$$

where the four-quark operators $Q_{1}$ and $Q_{2}$ are given by

$$
Q_{1}^{u^{\prime} d^{\prime}}=\bar{d}_{L}^{\prime} \gamma_{\mu} u_{L}^{\prime} \bar{c}_{L} \gamma^{\mu} b_{L}, \quad Q_{2}^{u^{\prime} d^{\prime}}=\bar{c}_{L} \gamma_{\mu} u_{L}^{\prime} \bar{d}_{L}^{\prime} \gamma^{\mu} b_{L}
$$

In the heavy-quark limit, the energy release is large, so the correlator in Eq. (5) is dominated by short-distance physics. An Operator Product Expansion (OPE) can be constructed for Eq. (5), which results in a prediction of decay widths of Eq. (5) as a series of matrix elements of local operators of increasing dimension suppressed by powers of $1 / m_{b}{ }^{1}$ :

$$
\Gamma\left(H_{b}\right)=\frac{1}{2 M_{H_{b}}} \sum_{k}\left\langle H_{b}\left|\mathcal{T}_{k}\right| H_{b}\right\rangle=\sum_{k} \frac{C_{k}(\mu)}{m_{b}^{k}}\left\langle H_{b}\left|\mathcal{O}_{k}^{\Delta B}=0(\mu)\right| H_{b}\right\rangle .
$$

In other words, the calculation of $\Gamma\left(H_{b}\right)$ is equivalent to computing the matching coefficients of the effective $\Delta B=0$ Lagrangian with subsequent computation of its matrix elements. Indeed, at the end, the scale dependence of the Wilson coefficients in Eq. (8) should match the scale dependence of the computed matrix elements.

It is customary to make predictions for the ratios of lifetimes (widths), as many theoretical uncertainties cancel out in the ratio. In addition, since the differences of lifetimes should come from the differences in the "brown mucks" of heavy hadrons, at the leading order in HQE all beauty hadrons with light spectators have the same lifetime.

The difference between meson and baryon lifetimes first occurs at order $1 / \mathrm{m}^{2}$ and is essentially due to the different structure of mesons and baryons. In other words, no lifetime difference is induced among the members of the meson multiplet. The ratio of heavy meson and baryon lifetimes receives a shift which amounts to at most $1-2 \%$, not sufficient to explain the observed pattern of lifetimes.

The main effect appears at the $1 / \mathrm{m}^{3}$ level and comes from the set of dimension-six fourquark operators. Their contribution is enhanced due to the phase-space factor $16 \pi^{2}$, and induces corrections of order $16 \pi^{2}\left(\Lambda_{Q C D} / m_{b}\right)^{3}=\mathcal{O}(5-10 \%)$. These operators introduce a difference in lifetimes for both heavy mesons and baryons. The effects of matrix elements of these operators,

\footnotetext{
${ }^{1}$ We choose a heavy-quark pole mass as an expansion parameter, which is consistent with most of the previous calculations. Of course, final predictions for the lifetime ratios are independent of that choice [8].
} 
commonly referred to as Weak Annihilation (WA), Weak Scattering (WS), and Pauli Interference (PI) have been computed in $[4,11,12,13,14,15,16]$ at leading order in perturbative QCD, and, more recently, including NLO perturbative QCD corrections in [6] and $1 / m_{b}$ corrections [7].

The contributions of these operators to the lifetime ratios are governed by the matrix elements of $\Delta B=0$ four-fermion operators

$$
\mathcal{T}_{\text {spec }}=\mathcal{T}_{\text {spec }}^{u}+\mathcal{T}_{\text {spec }}^{d^{\prime}}+\mathcal{T}_{\text {spec }}^{s^{\prime}},
$$

where the $\mathcal{T}_{i}$ are

$$
\begin{aligned}
\mathcal{T}_{\text {spec }}^{u}= & \frac{G_{F}^{2} m_{b}^{2}\left|V_{b c}\right|^{2}(1-z)^{2}}{2 \pi}\left\{\left(c_{1}^{2}+c_{2}^{2}\right) O_{1}^{u}+2 c_{1} c_{2} \widetilde{O}_{1}^{u}+\delta_{1 / m}^{u}+\delta_{1 / m^{2}}^{u}\right\}, \\
\mathcal{T}_{\text {spec }}^{d^{\prime}}= & -\frac{G_{F}^{2} m_{b}^{2}\left|V_{b c}\right|^{2}(1-z)^{2}}{4 \pi}\left\{c_{1}^{2}\left[(1+z) O_{1}^{d^{\prime}}+\frac{2}{3}(1+2 z) O_{2}^{d^{\prime}}\right]\right. \\
& \left.+\left(N_{c} c_{2}^{2}+2 c_{1} c_{2}\right)\left[(1+z) \widetilde{O}_{1}^{d^{\prime}}+\frac{2}{3}(1+2 z) \widetilde{O}_{2}^{d^{\prime}}\right]+\delta_{1 / m}^{d^{\prime}}+\delta_{1 / m^{2}}^{d^{\prime}}\right\}, \\
\mathcal{T}_{\text {spec }}^{s^{\prime}}= & -\frac{G_{F}^{2} m_{b}^{2}\left|V_{b c}\right|^{2} \sqrt{1-4 z}}{4 \pi}\left\{c_{1}^{2}\left[O_{1}^{s^{\prime}}+\frac{2}{3}(1+2 z) O_{2}^{s^{\prime}}\right]\right. \\
& \left.+\left(N_{c} c_{2}^{2}+2 c_{1} c_{2}\right)\left[\widetilde{O}_{1}^{s^{\prime}}+\frac{2}{3}(1+2 z) \widetilde{O}_{2}^{s^{\prime}}\right]+\delta_{1 / m}^{s^{\prime}}+\delta_{1 / m^{2}}^{s^{\prime}}\right\} .
\end{aligned}
$$

Here the terms $\delta_{1 / m}^{i}$ and $\delta_{1 / m^{2}}^{i}$ refer to $1 / m_{b}$ and $1 / m_{b}^{2}$ corrections to spectator effects, which we discuss below. Note that we include the full $z=m_{c}^{2} / m_{b}^{2}$ dependence, which is fully consistent only after the inclusion of higher $1 / m_{b}$ corrections. The operators $O_{i}$ and $\widetilde{O}_{i}$ in Eq. (10) are defined as

$$
\begin{array}{ll}
O_{1}^{q}=\bar{b}_{i} \gamma^{\mu}\left(1-\gamma_{5}\right) b_{i} \bar{q}_{j} \gamma_{\mu}\left(1-\gamma_{5}\right) q_{j}, & O_{2}^{q}=\bar{b}_{i} \gamma^{\mu} \gamma_{5} b_{i} \bar{q}_{j} \gamma_{\mu}\left(1-\gamma_{5}\right) q_{j}, \\
\widetilde{O}_{1}^{q}=\bar{b}_{i} \gamma^{\mu}\left(1-\gamma_{5}\right) b_{j} \bar{q}_{i} \gamma_{\mu}\left(1-\gamma_{5}\right) q_{j}, & \widetilde{O}_{2}^{q}=\bar{b}_{i} \gamma^{\mu} \gamma_{5} b_{j} \bar{q}_{i} \gamma_{\mu}\left(1-\gamma_{5}\right) q_{j} .
\end{array}
$$

In addition, there is a dimension- 6 operator $O_{3}^{q}=\bar{b}_{i} \gamma^{\mu}\left(1-\gamma_{5}\right) b_{i} \bar{q}_{j} \gamma_{\mu} \gamma_{5} q_{j}$ whose contribution is always suppressed by powers of the light-quark mass. It gives a negligible contribution to the ratios of lifetimes of heavy hadrons (see also Sect. 3).

In order to assess the impact of these and other operators, parametrizations of their matrix elements must be introduced. The meson matrix elements are

$$
\begin{aligned}
& \left\langle B_{q}\left|O_{1}^{q}\right| B_{q}\right\rangle=f_{B_{q}}^{2} m_{B_{q}}^{2}\left(2 \epsilon_{1}+\frac{B_{1}}{N_{c}}\right), \quad\left\langle B_{q}\left|\widetilde{O}_{1}^{q}\right| B_{q}\right\rangle=f_{B_{q}}^{2} m_{B_{q}}^{2} B_{1}, \\
& \left\langle B_{q}\left|O_{2}^{q}\right| B_{q}\right\rangle=-f_{B_{q}}^{2} m_{B_{q}}^{2}\left[\frac{m_{B_{q}}^{2}}{\left(m_{b}+m_{q}\right)^{2}}\left(2 \epsilon_{2}+\frac{B_{2}}{N_{c}}\right)+\frac{1}{2}\left(2 \epsilon_{1}+\frac{B_{1}}{N_{c}}\right)\right], \\
& \left\langle B_{q}\left|\widetilde{O}_{2}^{q}\right| B_{q}\right\rangle=-f_{B_{q}}^{2} m_{B_{q}}^{2}\left[\frac{m_{B_{q}}^{2}}{\left(m_{b}+m_{q}\right)^{2}} B_{2}+\frac{1}{2} B_{1}\right] .
\end{aligned}
$$

Here the parameters $B_{i}$ and $\epsilon_{i}$ are usually referred to as "singlet" and "octet" parameters (see Appendix A for the explanation). Expressed in terms of these parameters, the lifetime ratios of heavy 
mesons can be written as

$$
\begin{aligned}
\tau\left(B_{u}\right) / \tau\left(B_{d}\right)=1+16 \pi^{2} & \frac{f_{B}^{2} m_{B}}{m_{b}^{3} c_{3}\left(m_{b}\right)}\left[G_{1}^{s s}\left(m_{b}\right) B_{1}\left(m_{b}\right)+G_{1}^{o o}\left(m_{b}\right) \epsilon_{1}\left(m_{b}\right)\right. \\
& \left.+G_{2}^{s s}\left(m_{b}\right) B_{2}\left(m_{b}\right)+G_{1}^{o o}\left(m_{b}\right) \epsilon_{2}\left(m_{b}\right)\right]+\delta_{1 / m}
\end{aligned}
$$

where the coefficients $G$ were computed at NLO in $[15,6] . \delta_{1 / m}$ represents spectator corrections of order $1 / m_{b}$ and higher, which we present below in this paper.

Estimates of the matrix elements of four-fermion operators in baryon decays are not easy. Following [4], the parameter $\widetilde{B}$ is used to account for the deviation of the $\Lambda_{b}$ wave function from being totally color-asymmetric,

$$
\begin{aligned}
& \left\langle\Lambda_{b}\left|O_{1}^{q}\right| \Lambda_{b}\right\rangle=-\widetilde{B}\left\langle\Lambda_{b}\left|\widetilde{O}_{1}^{q}\right| \Lambda_{b}\right\rangle=\frac{\widetilde{B}}{6} f_{B_{q}}^{2} m_{B_{q}} m_{\Lambda_{b}} r \\
& \left\langle\Lambda_{b}\left|O_{2}^{q}\right| \Lambda_{b}\right\rangle=-\widetilde{B}\left\langle\Lambda_{b}\left|\widetilde{O}_{2}^{q}\right| \Lambda_{b}\right\rangle=\frac{\widetilde{B}}{6} f_{B_{q}}^{2} m_{B_{q}} m_{\Lambda_{b}} \delta
\end{aligned}
$$

Note that $\delta=\mathcal{O}\left(1 / m_{b}\right)$, which follows from the heavy-quark spin symmetry. It needs to be included as we consider higher-order corrections in $1 / m_{b} . \widetilde{B}=1$ in the valence approximation. While these parameters have not been computed model-independently, various quark-model arguments suggest that the meson and baryon matrix elements are quite different. Thus a meson-baryon lifetime difference can be produced. In general, one can parametrize the meson-baryon lifetime ratio as

$$
\begin{aligned}
\tau\left(\Lambda_{b}\right) / \tau\left(B_{d}\right) & \simeq 0.98-\left(d_{1}+d_{2} \widetilde{B}\right) r-\left(d_{3} \epsilon_{1}+d_{4} \epsilon_{2}\right)-\left(d_{5} B_{1}+d_{6} B_{2}\right) \\
& \simeq 0.98-m_{b}^{2}\left(d_{1}^{\prime}+d_{2}^{\prime} \widetilde{B}\right) r-m_{b}^{2}\left[\left(d_{3}^{\prime} \epsilon_{1}+d_{4}^{\prime} \epsilon_{2}\right)+\left(d_{5}^{\prime} B_{1}+d_{6}^{\prime} B_{2}\right)\right]
\end{aligned}
$$

where in the last line we scaled out the coefficient $m_{b}^{2}$ emphasizing the fact that these corrections are suppressed by $1 / m_{b}^{3}$ compared to the leading $m_{b}^{5}$ effect. The scale-dependent parameters $\left(d_{i}\left(m_{b}\right)=\{0.023,0.028,0 / 16,-0.16,0.08,-0.08\}\right.$ at NLO) are defined in [4]. The parameter $r=\left|\psi_{b q}^{\Lambda_{b}}(0)\right|^{2} /\left|\psi_{b \bar{q}}^{B_{q}}(0)\right|^{2}$ is the ratio of the wave functions at the origin of the $\Lambda_{b}$ and $B_{q}$ mesons.

It is interesting to note that in the absence of $1 / m_{b}$ corrections to spectator effects, it would be equally correct to substitute the $b$-quark mass in Eq. (15) with the corresponding meson and baryon masses, so

$$
\tau\left(\Lambda_{b}\right) / \tau\left(B_{d}\right) \simeq 0.98-m_{\Lambda_{b}}^{2}\left(d_{1}^{\prime}+d_{2}^{\prime} \widetilde{B}\right) r-m_{B_{d}}^{2}\left[\left(d_{3}^{\prime} \epsilon_{1}+d_{4}^{\prime} \epsilon_{2}\right)+\left(d_{5}^{\prime} B_{1}+d_{6}^{\prime} B_{2}\right)\right]
$$

which reflects the fact that WS and PI effects occur for the heavy and light quarks initially bound in the $B_{d}$ meson and $\Lambda_{b}$ baryon, respectively. While correct up to the order $1 / m_{b}^{3}$, these simple substitutions reduce the ratio of lifetimes by approximately $3-4 \%$ ! We take this as an indication of the importance of bound-state effects on the spectator corrections, represented by subleading $1 / m_{b}$ corrections to spectator operators. 

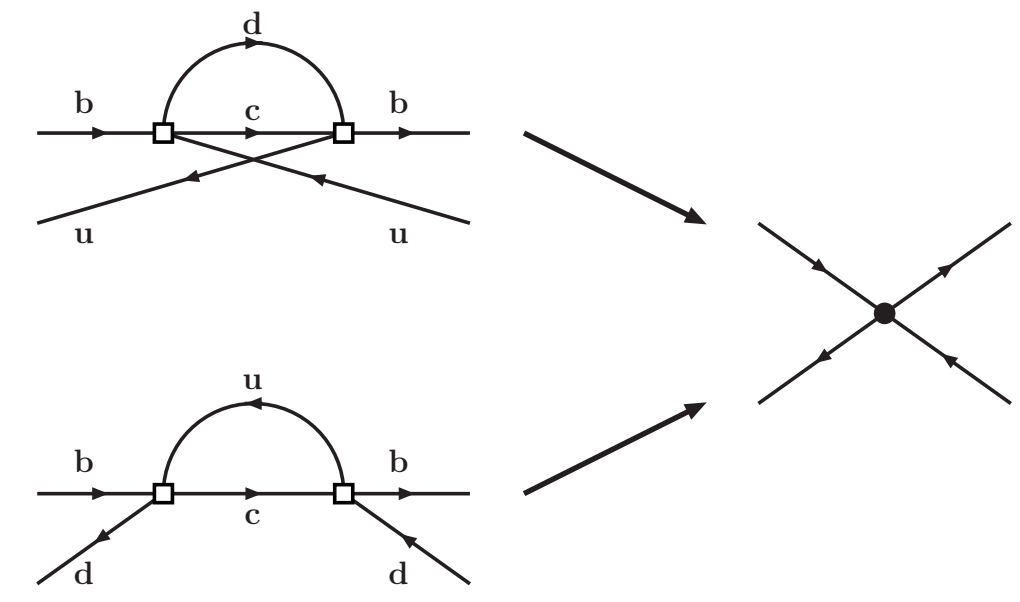

Figure 1: Kinetic corrections to spectator effects. The operators of Eqs. (17) and (19) are obtained by expanding the diagrams in powers of spectator's momentum.

\section{Subleading corrections to spectator effects}

We computed the higher order corrections, including charm quark-mass effects, to Eq. (10) in the heavy-quark expansion, denoted below as $\delta_{1 / m}^{q}$ and $\delta_{1 / m^{2}}^{q}$.

\section{3. $11 / m_{b}$ corrections}

The $1 / m_{b}$ corrections to the spectator effects are computed, as in Ref. [7], by expanding the forward scattering amplitude of Eq. (5) in the light-quark momentum and matching the result onto the operators containing derivative insertions (see Fig. 1). The $\delta_{1 / m}^{q}$ contributions can be written in the following form:

$$
\begin{aligned}
\delta_{1 / m}^{u}= & -2\left(c_{1}^{2}+c_{2}^{2}\right) \frac{1+z}{1-z} R_{1}^{u}-4 c_{1} c_{2} \frac{1+z}{1-z} \widetilde{R}_{1}^{u}, \\
\delta_{1 / m}^{d^{\prime}}= & c_{1}^{2}\left[\frac{8 z^{2}}{1-z} R_{0}^{d^{\prime}}+\frac{2}{3} \frac{1+z+10 z^{2}}{1-z} R_{1}^{d^{\prime}}+\frac{2}{3}(1+2 z)\left(R_{2}^{d^{\prime}}-R_{3}^{d^{\prime}}\right)\right], \\
& +\left(N_{c} c_{2}^{2}+2 c_{1} c_{2}\right)\left[\frac{8 z^{2}}{1-z} \widetilde{R}_{0}^{d^{\prime}}+\frac{2}{3} \frac{1+z+10 z^{2}}{1-z} \widetilde{R}_{1}^{d^{\prime}}+\frac{2}{3}(1+2 z)\left(\widetilde{R}_{2}^{d^{\prime}}-\widetilde{R}_{3}^{d^{\prime}}\right)\right], \\
\delta_{1 / m}^{s^{\prime}}= & c_{1}^{2}\left[\frac{16 z^{2}}{1-4 z} R_{0}^{s^{\prime}}+\frac{2}{3} \frac{1-2 z+16 z^{2}}{1-4 z} R_{1}^{s^{\prime}}+\frac{2}{3}(1+2 z)\left(R_{2}^{s^{\prime}}-R_{3}^{s^{\prime}}\right)\right] \\
& +\left(N_{c} c_{2}^{2}+2 c_{1} c_{2}\right)\left[\frac{16 z^{2}}{1-4 z} \widetilde{R}_{0}^{s^{\prime}}+\frac{2}{3} \frac{1-2 z+16 z^{2}}{1-4 z} \widetilde{R}_{1}^{s^{\prime}}+\frac{2}{3}(1+2 z)\left(\widetilde{R}_{2}^{s^{\prime}}-\widetilde{R}_{3}^{s^{\prime}}\right)\right],
\end{aligned}
$$


where the following operators contribute

$$
\begin{array}{ll}
R_{0}^{q}=\frac{1}{m_{b}^{2}} \bar{b}_{i} \gamma^{\mu} \gamma_{5} \vec{D}^{\alpha} b_{i} \bar{q}_{j} \gamma_{\mu}\left(1-\gamma_{5}\right) \vec{D}_{\alpha} q_{j}, & R_{1}^{q}=\frac{1}{m_{b}^{2}} \bar{b}_{i} \gamma^{\mu}\left(1-\gamma_{5}\right) \vec{D}^{\alpha} b_{i} \bar{q}_{j} \gamma_{\mu}\left(1-\gamma_{5}\right) \vec{D}_{\alpha} q_{j}, \\
R_{2}^{q}=\frac{1}{m_{b}^{2}} \bar{b}_{i} \gamma^{\mu}\left(1-\gamma_{5}\right) \vec{D}^{\nu} b_{i} \bar{q}_{j} \gamma_{\nu}\left(1-\gamma_{5}\right) \vec{D}_{\mu} q_{j}, & R_{3}^{q}=\frac{m_{q}}{m_{b}} \bar{b}_{i}\left(1-\gamma_{5}\right) b_{i} \bar{q}_{j}\left(1-\gamma_{5}\right) q_{j} .
\end{array}
$$

Here $\widetilde{R}_{i}^{q}$ denote the color-rearranged operators that follow from the expressions for $R_{i}^{q}$ by interchanging color indexes of $b_{i}$ and $q_{j}$ Dirac spinors. Note that the above result contains full QCD $b$-fields, thus there is no immediate power counting available for these operators. The power counting becomes manifest at the level of the matrix elements.

In the $z \rightarrow 0$ limit the result of Eq. (17) reduces to the set of $1 / m_{b}$ corrections obtained by us in Ref. [7]. We complete the calculation presented there by computing the contribution of Eq. (17) to the ratios of heavy-meson lifetimes. As before, we use factorization to guide our parametrizations of $\Lambda_{b}$ and meson matrix elements, but, contrary to Ref. [7], we elect to keep corrections that would vanish in the vacuum-insertion approximation. Our parametrizations for meson and baryon matrix elements are presented in Appendix B. Inserting Eqs. (B.2) and (B.5) of Appendix B into Eq. (17) we obtain an estimate of the effect. We postpone the presentation of the answer, complete with our estimate of its uncertainties, until the end of this section, after we assess the convergence of the $1 / m_{b}$ expansion for the spectator corrections.

Numerically, the set of $1 / m_{b}$ corrections does not markedly affect the ratios of meson lifetimes, changing the $\tau\left(B_{u}\right) / \tau\left(B_{d}\right)$ and the $\tau\left(B_{s}\right) / \tau\left(B_{d}\right)$ ratios by less than half a percent. The effect is more pronounced in the ratio of $\Lambda_{b}$ and $B_{d}$ lifetimes, where it constitutes a $40-45 \%$ of the leading spectator contribution represented by WS and PI effects (see Eqs. $(15,16)$ ), or an overall correction of about $-(3-4) \%$ to the $\tau\left(\Lambda_{b}\right) / \tau\left(B_{d}\right)$ ratio. While such a sizable effect is surprising, the main source of such a large correction can be readily identified. While the individual $1 / m_{b}$ corrections to the matrix elements representing WS and PI are of order 20\%, as expected from the naive power counting, they contribute to the $\Lambda_{b}$ lifetime with the same (negative) sign, instead of destructively interfering as do the contributions to Eqs. $(15,16)$ representing WS and PI [5], as explained in [7]. This conspiracy of two $\sim 20 \%$ effects produces a sizable shift in the ratio of the $\Lambda_{b}$ and $B$-meson lifetimes.

\section{3. $21 / m_{b}^{2}$ corrections}

In order to see how well the $1 / m_{b}$ expansion converges in the calculation of lifetime ratios, we compute a set of $\delta_{1 / m_{b}^{2}}$ corrections to spectator effects. It is expected that at this order even more operators will contribute, so the exact prediction of lifetime differences becomes unfeasible. Therefore, we shall use our calculation only to see to what extent we can trust our results for the $1 / m_{b}$ corrections. We will parametrize $1 / m_{b}^{2}$ corrections similar to our parametrization of $1 / m_{b}$ effects above and will use the factorization approximation to assess their contributions to the lifetime ratios.

There are two classes of corrections that arise at this order. One class involves kinetic corrections which can be computed in a way analogous to the previous case by expanding the forward 
scattering amplitudes in the powers of spectator momentum (see Fig. 1). A second class involves corrections arising from an interaction with background gluon fields. The kinetic corrections can be written as:

$$
\begin{aligned}
& \delta_{1 / m^{2}}^{u}=\left(c_{1}^{2}+c_{2}^{2}\right)\left[\frac{m_{u}^{2}}{m_{b}^{2}} \frac{1+z}{1-z} O_{1}^{u}+\frac{8 z^{2}}{(1-z)^{2}} W_{1}^{u}\right]+2 c_{1} c_{2}\left[\frac{m_{u}^{2}}{m_{b}^{2}} \frac{1+z}{1-z} \widetilde{O}_{1}^{u}+\frac{8 z^{2}}{(1-z)^{2}} \widetilde{W}_{1}^{u}\right], \\
& \delta_{1 / m^{2}}^{d^{\prime}}=c_{1}^{2}\left[\frac{m_{d^{\prime}}^{2}}{m_{b}^{2}} \frac{1+z+2 z^{2}}{1-z} O_{1}^{d^{\prime}}+\frac{m_{d^{\prime}}^{2}}{m_{b}^{2}} \frac{4 z^{2}}{1-z} O_{2}^{d^{\prime}}+\frac{m_{d^{\prime}}^{2}}{m_{b}^{2}} \frac{2(1+2 z)}{3} O_{3}^{d^{\prime}}\right. \\
& \left.+\frac{4 z^{2}(7 z-5)}{(1-z)^{2}} W_{1}^{d^{\prime}}+\frac{8 z^{2}(4 z-3)}{(1-z)^{2}} W_{2}^{d^{\prime}}+\frac{8 z^{2}}{1-z}\left(W_{3}^{d^{\prime}}-W_{4}^{d^{\prime}}\right)\right] \\
& +\left(N_{c} c_{2}^{2}+c_{1} c_{2}\right)\left[\frac{m_{d^{\prime}}^{2}}{m_{b}^{2}} \frac{1+z+2 z^{2}}{1-z} \widetilde{O}_{1}^{d^{\prime}}+\frac{m_{d^{\prime}}^{2}}{m_{b}^{2}} \frac{4 z^{2}}{1-z} \widetilde{O}_{2}^{d^{\prime}}+\frac{m_{d^{\prime}}^{2}}{m_{b}^{2}} \frac{2(1+2 z)}{3} \widetilde{O}_{3}^{d^{\prime}}\right. \\
& \left.+\frac{4 z^{2}(7 z-5)}{(1-z)^{2}} \widetilde{W}_{1}^{d^{\prime}}+\frac{8 z^{2}(4 z-3)}{(1-z)^{2}} \widetilde{W}_{2}^{d^{\prime}}+\frac{8 z^{2}}{1-z}\left(\widetilde{W}_{3}^{d^{\prime}}-\widetilde{W}_{4}^{d^{\prime}}\right)\right] \\
& \delta_{1 / m^{2}}^{s^{\prime}}=c_{1}\left[\frac{m_{s^{\prime}}^{2}}{m_{b}^{2}} \frac{1-2 z}{1-4 z} O_{1}^{s^{\prime}}+\frac{m_{s^{\prime}}^{2}}{m_{b}^{2}} \frac{8 z^{2}}{1-4 z} O_{2}^{s^{\prime}}+\frac{m_{s^{\prime}}^{2}}{m_{b}^{2}} \frac{2(1+2 z)}{3} O_{3}^{s^{\prime}}\right. \\
& \left.+\frac{8 z^{2}(16 z-5)}{(1-4 z)^{2}} W_{1}^{s^{\prime}}+\frac{16 z^{2}(10 z-3)}{(1-4 z)^{2}} W_{2}^{s^{\prime}}+\frac{16 z^{2}}{1-4 z}\left(W_{3}^{s^{\prime}}-W_{4}^{s^{\prime}}\right)\right] \\
& +\left(N_{c} c_{2}^{2}+2 c_{1} c_{2}\right)\left[\frac{m_{s^{\prime}}^{2}}{m_{b}^{2}} \frac{1-2 z}{1-4 z} \widetilde{O}_{1}^{s^{\prime}}+\frac{m_{s^{\prime}}^{2}}{m_{b}^{2}} \frac{8 z^{2}}{1-4 z} \widetilde{O}_{2}^{s^{\prime}}+\frac{m_{s^{\prime}}^{2}}{m_{b}^{2}} \frac{2(1+2 z)}{3} \widetilde{O}_{3}^{s^{\prime}}\right. \\
& \left.+\frac{8 z^{2}(16 z-5)}{(1-4 z)^{2}} \widetilde{W}_{1}^{s^{\prime}}+\frac{16 z^{2}(10 z-3)}{(1-4 z)^{2}} \widetilde{W}_{2}^{s^{\prime}}+\frac{16 z^{2}}{1-4 z}\left(\widetilde{W}_{3}^{s^{\prime}}-\widetilde{W}_{4}^{s^{\prime}}\right)\right] .
\end{aligned}
$$

We again retain the dependence on quark masses in the above expression, including the terms proportional to light-quark masses (and thus multiplied by the operators $O_{i}$ ). The operators in Eq. (19) are defined as

$$
\begin{aligned}
& W_{1}^{q}=\frac{1}{m_{b}^{4}} \bar{b}_{i} \gamma^{\mu}\left(1-\gamma_{5}\right) \vec{D}^{\alpha} \vec{D}^{\beta} b_{i} \bar{q}_{j} \gamma_{\mu}\left(1-\gamma_{5}\right) \vec{D}_{\alpha} \vec{D}_{\beta} q_{j}, \quad W_{2}^{q}=\frac{1}{m_{b}^{4}} \bar{b}_{i} \gamma^{\mu} \gamma_{5} \vec{D}^{\alpha} \vec{D}^{\beta} b_{i} \bar{q}_{j} \gamma_{\mu}\left(1-\gamma_{5}\right) \vec{D}_{\alpha} \vec{D}_{\beta} q_{j}, \\
& W_{3}^{q}=\frac{1}{m_{b}^{4}} \bar{b}_{i} \gamma^{\mu}\left(1-\gamma_{5}\right) \vec{D}^{\nu} \vec{D}^{\alpha} b_{i} \bar{q}_{j} \gamma_{\nu}\left(1-\gamma_{5}\right) \vec{D}_{\mu} \vec{D}_{\alpha} q_{j}, \quad W_{4}^{q}=\frac{m_{q}}{m_{b}} \bar{b}_{i}\left(1-\gamma_{5}\right) \vec{D}^{\alpha} b_{i} \bar{q}_{j}\left(1-\gamma_{5}\right) \vec{D}_{\alpha} q_{j},
\end{aligned}
$$

where, as before, $\widetilde{W}_{i}^{q}$ denote the color-rearranged operators that follow from the expressions for $W_{i}^{q}$ by interchanging the color indexes of $b_{i}$ and $q_{j}$ Dirac spinors. The parametrization of matrix elements of these operators can be found in Appendix B.

In addition to the set of kinetic corrections considered above, the effects of the interactions of the intermediate quarks with background gluon fields should also be included at this order. The contribution of those operators can be computed from the diagrams of Fig. 2, resulting in

$$
\mathcal{T}_{\text {spec }, G}=\mathcal{T}_{\text {spec }, G}^{u}+\mathcal{T}_{\text {spec }, G}^{d^{\prime}}+\mathcal{T}_{\text {spec }, G}^{s^{\prime}}
$$



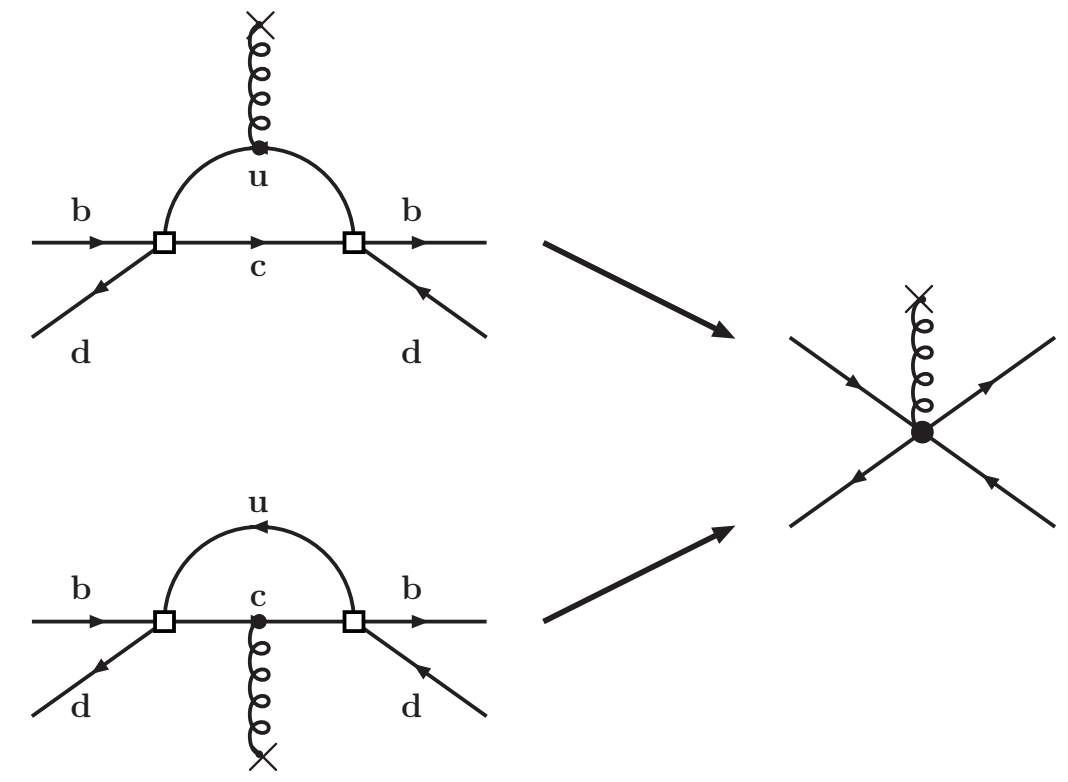

Figure 2: Corrections to spectator contribution coming from the interactions with background gluon fields.

where

$$
\begin{aligned}
\mathcal{T}_{\text {spec }, G}^{u}= & 0, \\
\mathcal{T}_{\text {spec }, G}^{d^{\prime}}= & -\frac{G_{F}^{2}\left|V_{b c}\right|^{2}}{4 \pi}\left\{c_{1}^{2}\left[\left(1-z^{2}\right) P_{1}^{d^{\prime}}-\left(1-z^{2}\right) P_{2}^{d^{\prime}}+2 z(1-z) P_{3}^{d^{\prime}}+4 z^{2} P_{4}^{d^{\prime}}\right]\right. \\
& \left.+2 c_{1} c_{2} z\left[(1-z) P_{5}^{d^{\prime}}+(1-z) P_{6}^{d^{\prime}}+2 z P_{7}^{d^{\prime}}+2 z P_{8}^{d^{\prime}}\right]\right\}, \\
\mathcal{T}_{\text {spec }, G}^{s^{\prime}}= & -\frac{G_{F}^{2}\left|V_{b c}\right|^{2}}{4 \pi \sqrt{1-4 z}}\left\{c_{1}^{2}\left[(1-2 z) P_{1}^{s^{\prime}}-(1-4 z) P_{2}^{s^{\prime}}+2 z P_{3}^{s^{\prime}}+4 z P_{4}^{s^{\prime}}\right]\right. \\
& \left.+4 c_{1} c_{2} z\left[P_{7}^{s^{\prime}}+P_{8}^{s^{\prime}}+P_{9}^{s^{\prime}}+P_{10}^{s^{\prime}}\right]\right\} .
\end{aligned}
$$

The local four-quark operators in the above formulas are shown in Eq. (25).

$$
\begin{aligned}
& P_{1}^{q}=\bar{b}_{k} \gamma^{\mu}\left(1-\gamma_{5}\right) t_{k l}^{a} \widetilde{G}_{\mu \nu}^{a} b_{l} \bar{q}_{i} \gamma^{\nu}\left(1-\gamma_{5}\right) q_{i}, \\
& P_{2}^{q}=\bar{b}_{i} \gamma^{\mu}\left(1-\gamma_{5}\right) b_{i} \bar{q}_{k} \gamma^{\nu}\left(1-\gamma_{5}\right) t_{k l}^{a} \widetilde{G}_{\mu \nu}^{a} q_{l}, \\
& P_{3}^{q}=\bar{b}_{k} \gamma^{\mu}\left(1+\gamma_{5}\right) t_{k l}^{a} \widetilde{G}_{\mu \nu}^{a} b_{l} \bar{q}_{i} \gamma^{\nu}\left(1-\gamma_{5}\right) q_{i}, \\
& P_{4}^{q}=\frac{1}{m_{b}^{2}} \bar{b}_{i} \gamma^{\mu}\left(1-\gamma_{5}\right) \vec{D}^{\nu} \vec{D}^{\alpha} b_{i} \bar{q}_{k} \gamma_{\alpha}\left(1-\gamma_{5}\right) t_{k l}^{a} \widetilde{G}_{\mu \nu}^{a} q_{l}, \\
& P_{5}^{q}=\bar{b}_{k} \gamma^{\mu}\left(1+\gamma_{5}\right) b_{i} t_{k l}^{a} \widetilde{G}_{\mu \nu}^{a} \bar{q}_{i} \gamma^{\nu}\left(1-\gamma_{5}\right) q_{l}, \\
& P_{6}^{q}=\bar{b}_{i} \gamma^{\mu}\left(1+\gamma_{5}\right) b_{k} t_{k l}^{a} \widetilde{G}_{\mu \nu}^{a} \bar{q}_{l} \gamma^{\nu}\left(1-\gamma_{5}\right) q_{i},
\end{aligned}
$$




$$
\begin{aligned}
P_{7}^{q} & =\frac{1}{m_{b}^{2}} \bar{b}_{k} \gamma^{\mu}\left(1-\gamma_{5}\right) \vec{D}^{\nu} \vec{D}^{\alpha} b_{i} t_{k l}^{a} \widetilde{G}_{\mu \nu}^{a} \bar{q}_{i} \gamma_{\alpha}\left(1-\gamma_{5}\right) q_{l}, \\
P_{8}^{q} & =\frac{1}{m_{b}^{2}} \bar{b}_{i} \gamma^{\mu}\left(1-\gamma_{5}\right) \vec{D}^{\nu} \vec{D}^{\alpha} b_{k} t_{k l}^{a} \widetilde{G}_{\mu \nu}^{a} \bar{q}_{l} \gamma_{\alpha}\left(1-\gamma_{5}\right) q_{i}, \\
P_{9}^{q} & =\bar{b}_{k} \gamma^{\mu} b_{i} t_{k l}^{a} \widetilde{G}_{\mu \nu}^{a} \bar{q}_{i} \gamma^{\nu}\left(1-\gamma_{5}\right) q_{l}, \\
P_{10}^{q} & =\bar{b}_{i} \gamma^{\mu} b_{k} t_{k l}^{a} G_{\mu \nu}^{a} \bar{q}_{l} \gamma^{\nu}\left(1-\gamma_{5}\right) q_{i} .
\end{aligned}
$$

Analogously to the previous section, we parametrize the matrix elements as

$$
\left\langle B_{q}\left|P_{i}^{d^{\prime}}\right| B_{q}\right\rangle=\frac{1}{4} f_{B_{q}}^{2} m_{B_{q}}^{2} \alpha_{i}^{q}\left(\frac{m_{B_{q}}^{2}}{m_{b}^{2}}-1\right)^{2},\left\langle\Lambda_{b}\left|P_{i}^{q}\right| \Lambda_{b}\right\rangle=\frac{1}{4} f_{B_{q}}^{2} m_{B_{q}} m_{\Lambda_{b}} \alpha_{i}^{\Lambda}\left(\frac{m_{\Lambda_{b}}^{2}}{m_{b}^{2}}-1\right)^{2} .
$$

We set $\alpha_{i}^{q}=\alpha_{i}^{\Lambda}=1 \mathrm{GeV}^{2}$ to obtain a numerical estimate of this effect. It is clear that no precise prediction is possible with so many operators contributing to the ratios of lifetimes. This, of course, is expected, as the number of contributing operators always increase significantly with each order in OPE. Fortunately, at least in factorization, the effect of $1 / m_{b}^{2}$ corrections is tiny, a fraction of a percent. The contribution of $1 / m_{b}^{2}$-suppressed effect is included into the coefficient $k_{0}$ defined in the next section. We can evaluate the contribution of both $1 / m_{b}$ and $1 / m_{b}^{2}$ by performing two analyses. First, we can study the perturbative scale dependence of the final result using "factorized" values for the matrix elements ${ }^{2}$. We shall see that the scale dependence of the resulting effect is very mild. Second, we shall randomly vary the parameters describing the matrix elements by $\pm 30 \%$ around their "factorized" values to see the uncertainty of our result.

\section{3 Phenomenology of heavy hadron lifetimes}

Let us now discuss the phenomenological implications of the results presented in the previous sections. As usual in the OPE-based calculation, next-order corrections bring new unknown coefficients. In our numerical results we assume the value of the $b$-quark pole mass to be $m_{b}=$ $4.8 \pm 0.1 \mathrm{GeV}$ and $f_{B}=200 \pm 25 \mathrm{MeV}$.

The resulting lifetime ratios are parametrized according to Appendix B. For instance, in the case of $\tau\left(\Lambda_{b}\right) / \tau\left(B_{d}\right)$ we can write

$$
\left.\frac{\tau\left(\Lambda_{b}\right)}{\tau\left(B_{d}\right)}\right|_{h . o .}=k_{0}+\sum_{i=1} k_{i} p_{i}
$$

where $p_{i}$ are the parameters defined in Appendix B,

$$
p_{i}=\left\{B_{1}, B_{2}, \epsilon_{1}, \epsilon_{2}, r, \delta, \widetilde{B} r, \widetilde{B} \delta, \beta_{0}, \widetilde{\beta}_{0}, \beta_{1}, \widetilde{\beta}_{1}, \beta_{2}, \widetilde{\beta}_{2}, \beta_{3}, \widetilde{\beta}_{3}, \beta_{0}^{\Lambda}, \widetilde{\beta}_{0}^{\Lambda}, \beta_{1}^{\Lambda}, \widetilde{\beta}_{1}^{\Lambda}, \beta_{2}^{\Lambda}, \widetilde{\beta}_{2}^{\Lambda}, \beta_{3}^{\Lambda}, \widetilde{\beta}_{3}^{\Lambda}\right\},
$$

\footnotetext{
${ }^{2}$ In the absence of model-independent calculations of the matrix elements described above, there is a number of ways to estimate them. We chose to use the quark model to relate the matrix elements of $R_{i}$ and $\widetilde{R}_{i}$ operators to the matrix elements of dimension 6 operators $O_{i}$, which are parametrized in terms of $B_{i}$ and $\epsilon_{i}$. We then use lattice QCD predictions for $B_{i}$ and $\epsilon_{i}$ to obtain numerical estimates of our corrections. We shall refer to this procedure as "factorization."
} 


\begin{tabular}{|c|cccccccccccc|}
\hline \hline & \multicolumn{10}{|c|}{ Coefficients $k_{i}$ for the parameters listed below. All entries are $\times 10^{-3}}$. \\
$\mu$ & $B_{1}$ & $B_{2}$ & $\epsilon_{1}$ & $\epsilon_{2}$ & $r$ & $\delta$ & $\widetilde{B} r$ & $\widetilde{B} \delta$ & $\beta_{0}$ & $\widetilde{\beta}_{0}$ & $\beta_{1}$ & $\widetilde{\beta}_{1}$ \\
\hline$m_{b} / 2$ & -7.82 & 9.19 & -158 & 183 & -27.4 & 3.76 & -33.3 & 11.1 & 0.001 & 1.95 & -0.014 & -24.0 \\
$m_{b}$ & -7.96 & 9.31 & -160 & 196 & -22.4 & 3.39 & -27.8 & 12.4 & 0.031 & 1.78 & -0.382 & -21.9 \\
$2 m_{b}$ & -7.45 & 8.63 & -160 & 201 & -18.9 & 3.33 & -24.6 & 13.0 & 0.079 & 1.69 & -0.976 & -20.9 \\
\hline \hline$\mu$ & $\beta_{2}$ & $\widetilde{\beta}_{2}$ & $\beta_{3}$ & $\widetilde{\beta}_{3}$ & $\beta_{0}^{\Lambda}$ & $\widetilde{\beta}_{0}^{\Lambda}$ & $\beta_{1}^{\Lambda}$ & $\widetilde{\beta}_{1}^{\Lambda}$ & $\beta_{2}^{\Lambda}$ & $\widetilde{\beta}_{2}^{\Lambda}$ & $\beta_{3}^{\Lambda}$ & $\widetilde{\beta}_{3}^{\Lambda}$ \\
\hline$m_{b} / 2$ & -0.002 & -3.68 & 0.002 & 3.68 & -0.144 & -0.048 & -12.7 & -6.85 & -0.818 & -0.272 & -0.818 & -0.272 \\
$m_{b}$ & -0.058 & -3.36 & 0.058 & 3.36 & -0.132 & -0.039 & -11.2 & -4.59 & -0.747 & -0.223 & -0.747 & -0.223 \\
$2 m_{b}$ & -0.150 & -3.20 & 0.150 & 3.20 & -0.125 & -0.030 & -10.4 & -3.09 & -0.711 & -0.170 & -0.711 & -0.170 \\
\hline \hline
\end{tabular}

Table 1: Coefficients $k_{i}$ appearing in Eq. (27) for $\tau\left(\Lambda_{b}\right) / \tau\left(B_{d}\right)$.

\begin{tabular}{|c|cccccccccccc|}
\hline \hline & \multicolumn{10}{|c|}{ Coefficients $k_{i}$ for the parameters listed below. All entries are $\times 10^{-3}}$. \\
$\mu$ & $B_{1}$ & $B_{2}$ & $\epsilon_{1}$ & $\epsilon_{2}$ & $\beta_{0}$ & $\widetilde{\beta}_{0}$ & $\beta_{1}$ & $\widetilde{\beta}_{1}$ & $\beta_{2}$ & $\widetilde{\beta}_{2}$ & $\beta_{3}$ & $\widetilde{\beta}_{3}$ \\
\hline$m_{b} / 2$ & 82.1 & 18.8 & -782 & 206 & 0.001 & 1.95 & -17.6 & 124 & -0.002 & -3.68 & 0.002 & 3.68 \\
$m_{b}$ & 60.8 & 13.3 & -727 & 211 & 0.031 & 1.78 & -6.58 & 107 & -0.058 & -3.36 & 0.058 & 3.36 \\
$2 m_{b}$ & 46.5 & 9.98 & -693 & 213 & 0.079 & 1.69 & 0.661 & 98.9 & -0.150 & -3.20 & 0.150 & 3.20 \\
\hline \hline
\end{tabular}

Table 2: Same as Table 1 for $\tau\left(B_{u}\right) / \tau\left(B_{d}\right)$.

and $k_{i}$ are their coefficients. $k_{0}=1.00$ in the case of mesons, while for $\Lambda_{b} k_{0}=\{0.991,0.981,0.975\}$ for $\mu=\left\{m_{b} / 2, m_{b}, 2 m_{b}\right\}$, respectively. They appear in Table 1, and a similar procedure is followed for $\tau\left(B_{u}\right) / \tau\left(B_{d}\right)$ and $\tau\left(B_{s}\right) / \tau\left(B_{d}\right)$, whose coefficients are shown in Tables 2 and 3, respectively.

Since all three heavy mesons belong to the same $S U(3)$ triplet, their lifetimes are the same at order $1 / m_{b}^{2}$. The computation of the ratios of heavy meson lifetimes is equivalent to the computation of $U$-spin or isospin-violating corrections. Both $1 / m_{b}^{3}$-suppressed spectator effects and our corrections computed in the previous sections arise from the spectator interactions and thus provide a source of $U$-spin or isospin-symmetry breaking. We shall, however, assume that the matrix elements of both $1 / m_{b}^{3}$ and $1 / m_{b}^{4}$ operators respect isospin. The ratio of lifetimes of $B_{s}$ and $B_{d}$ mesons involves a breaking of $U$-spin symmetry, so the matrix elements of dimension- 6 operators could differ by about $30 \%$. We partially take this breaking into account by retaining light quark-mass dependence. Nevertheless, we still introduce different $B-$ and $\epsilon$-parameters to describe $B_{s}$ and $B_{d}$ lifetimes. We will also assume that, apart from the explicit light quark-mass effects, matrix elements of $1 / m_{b}^{4}$ operators respect $U$-spin symmetry.

Since the computation of $1 / m_{b}$ corrections to spectator effects was performed at leading order in QCD, we also studied the scale dependence of the result. A comparison between the results computed with leading logarithmic accuracy (LL), next-to-leading log accuracy (NLL) and the total NLL including $1 / m_{b}$ and $1 / m_{b}^{2}$ corrections is plotted for $\tau\left(\Lambda_{b}\right) / \tau\left(B_{d}\right)$ in Fig. 3 for a renormalization scale parameter interval $m_{b} / 2 \leq \mu \leq 2 m_{b}$, assuming "factorization", i.e. setting all the $\beta$-parameters in Eqs. (B.2), (B.5) to the value calculated using our "factorization" ansatz. Figs. 4 and 5 show analogous plots for $\tau\left(B_{u}\right) / \tau\left(B_{d}\right)$ and $\tau\left(B_{s}\right) / \tau\left(B_{d}\right)$. As can be seen from these graphs, 


\begin{tabular}{|c|cccccccc|}
\hline \hline & \multicolumn{8}{|c|}{ Coefficients $k_{i}$ for the parameters listed below. All entries are $\times 10^{-3}$. } \\
$\mu$ & $B_{1}$ & $B_{2}$ & $\epsilon_{1}$ & $\epsilon_{2}$ & $B_{1}^{s}$ & $B_{2}^{s}$ & $\epsilon_{1}^{s}$ & $\epsilon_{2}^{s}$ \\
\hline$m_{b} / 2$ & -7.82 & 9.19 & -158 & 184 & 11.2 & -12.4 & 180 & -230 \\
$m_{b}$ & -7.96 & 9.31 & -160 & 196 & 13.5 & -16.8 & 182 & -243 \\
$2 m_{b}$ & -7.45 & 8.63 & -160 & 201 & 14.7 & -19.1 & 183 & -249 \\
\hline \hline$\mu$ & $\beta_{0}$ & $\widetilde{\beta}_{0}$ & $\beta_{1}$ & $\widetilde{\beta}_{1}$ & $\beta_{2}$ & $\widetilde{\beta}_{2}$ & $\beta_{3}$ & $\widetilde{\beta}_{3}$ \\
\hline$m_{b} / 2$ & -0.003 & -5.72 & 0.011 & 18.9 & 0.0013 & 2.20 & -0.0013 & -2.20 \\
$m_{b}$ & -0.091 & -5.23 & 0.300 & 17.3 & 0.0349 & 2.00 & -0.0349 & -2.00 \\
$2 m_{b}$ & -0.233 & -4.97 & 0.768 & 16.4 & 0.0892 & 1.91 & -0.0892 & -1.91 \\
\hline \hline
\end{tabular}

Table 3: Same as Table 1 for $\tau\left(B_{s}\right) / \tau\left(B_{d}\right)$.

the scale dependence of the predictions is very mild. Next, we fix the scale in our calculations

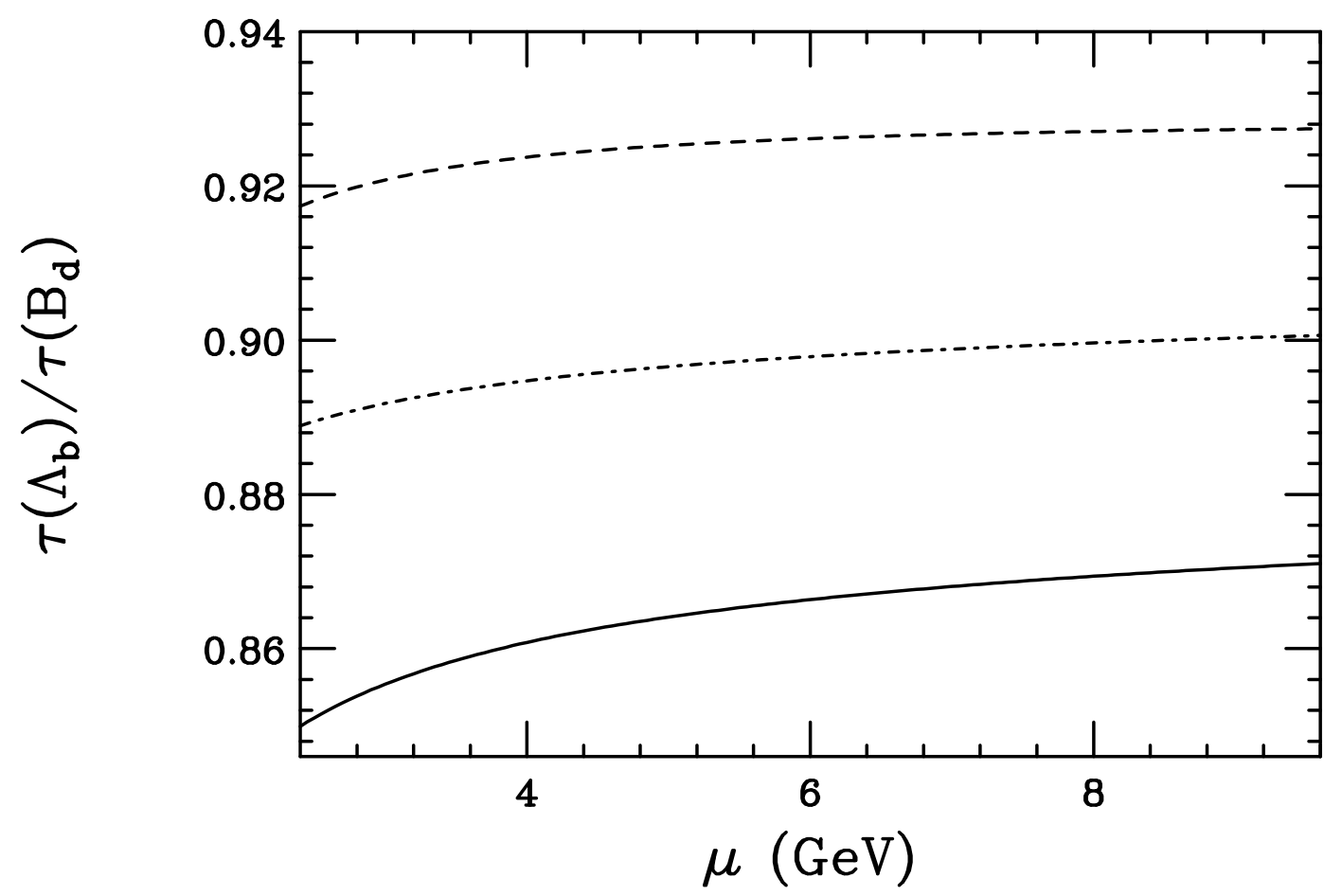

Figure 3: LL (dashed line), NLL (dash-dotted line), and NLL $+1 / m_{b}$ (solid line) contributions to $\tau\left(\Lambda_{b}\right) / \tau\left(B_{d}\right)$ are plotted vs. the scale parameter $\mu$ in $\mathrm{GeV}$.

and vary the values of parameters of the matrix elements. We adopt the statistical approach for presenting our results and generate 20000-point probability distributions of the ratios of lifetimes obtained by randomly varying our parameters within a $\pm 30 \%$ interval around their "factorization" values, for three different scales $\mu$. The decay constants $f_{B_{q}}$ and b-quark pole mass $m_{b}$ are taken to vary within $1 \sigma$ interval indicated above. The results are presented in Figs. 6, 7, and 8. 


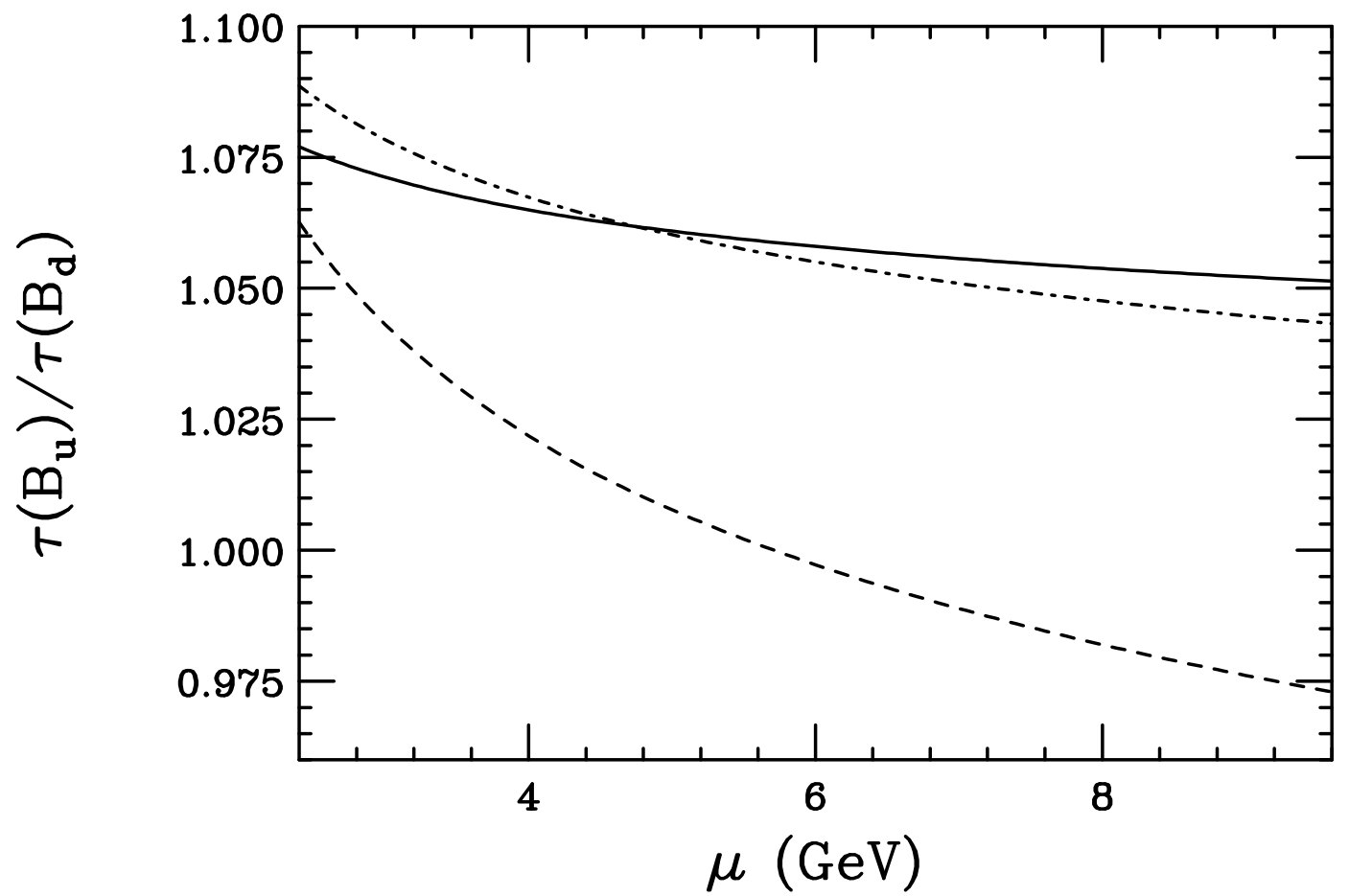

Figure 4: Same as Fig. 3 for $\tau\left(B_{u}\right) / \tau\left(B_{d}\right)$.

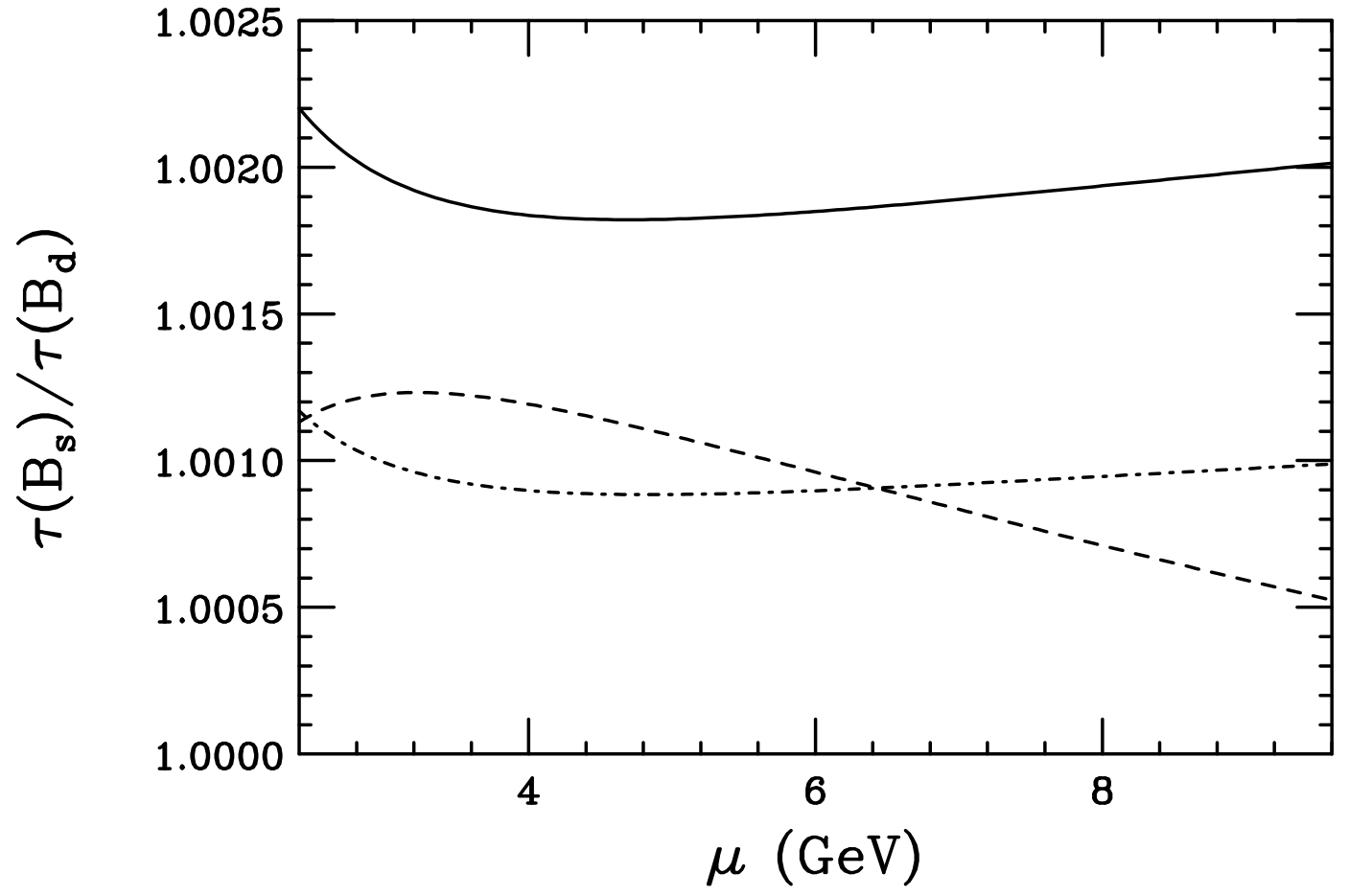

Figure 5: Same as Fig. 3 for $\tau\left(B_{s}\right) / \tau\left(B_{d}\right)$. 
These figures represent the main result of this paper. They show a nice agreement between the

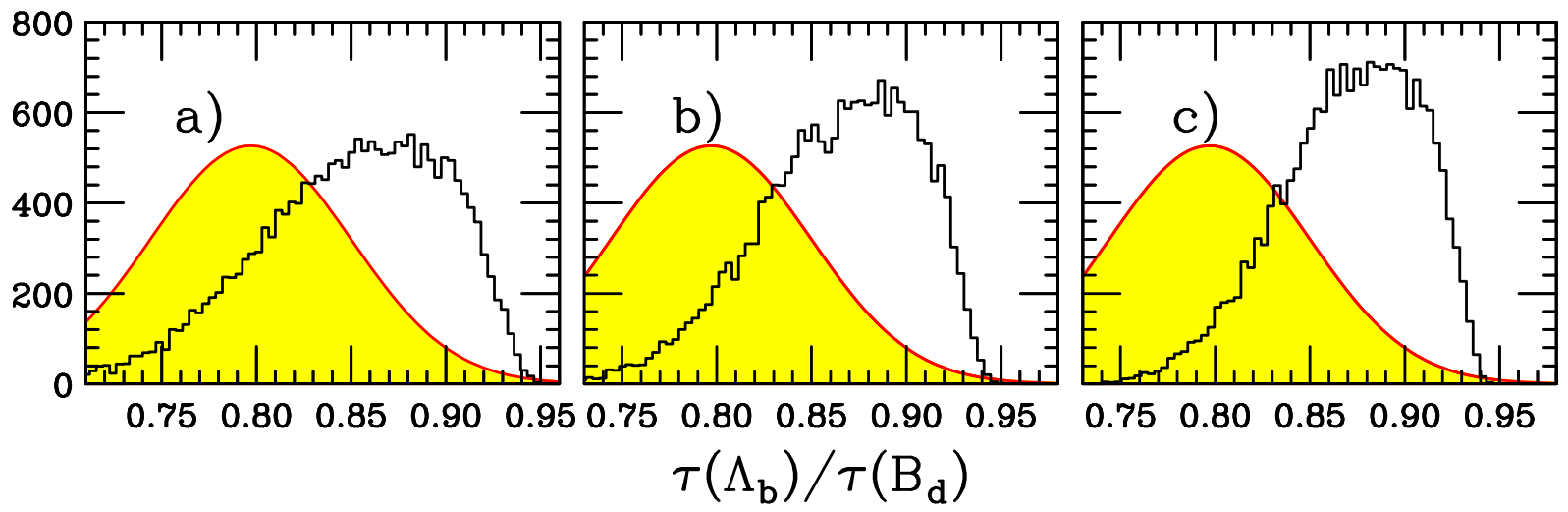

Figure 6: Histograms showing the random distributions around the central values of the $f_{B_{q}}, m_{b}, B, \delta, \epsilon, \beta_{i}$ and $\widetilde{\beta}_{i}$ parameters of Eqs. (B.2) and (B.5) contributing to $\tau\left(\Lambda_{b}\right) / \tau\left(B_{d}\right)$. Three histograms are shown for the scales $\mu=$ $m_{b} / 2$ (a), $\mu=m_{b}(\mathrm{~b})$, and $\mu=2 m_{b}$ (c). The shaded curves, presented for the convenience, represent current experimental result. Their normalization is arbitrary.

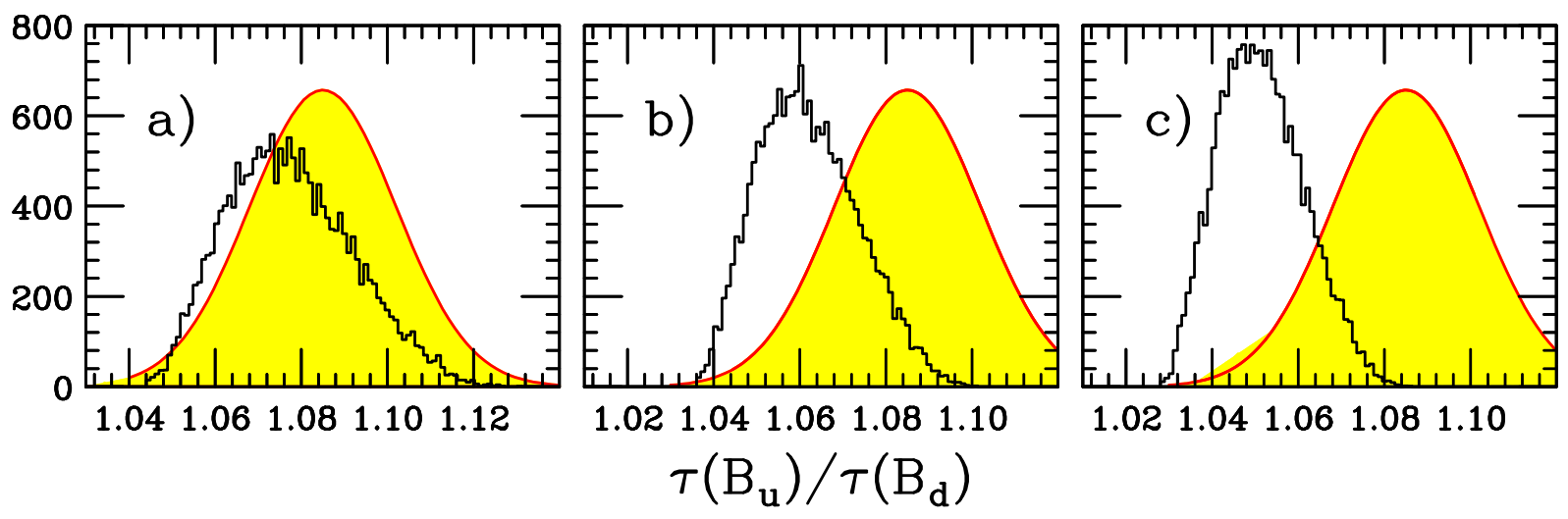

Figure 7: Same as Fig. 6 for $\tau\left(B_{u}\right) / \tau\left(B_{d}\right)$.

experimental results and theoretical predictions for all of the ratios of lifetimes of heavy hadrons. In fact, it is a relatively "large" ratio of $\tau\left(B_{s}\right) / \tau\left(B_{d}\right)$ that can pose a potential problem if future measurements at Fermilab and CERN would find the mean value to stay the same with its error bars shrinking. Upcoming Fermilab and CERN measurements would again clarify this issue.

It also appears that the theoretical errors in the ratio of $\tau\left(B_{s}\right) / \tau\left(B_{d}\right)$ are much smaller than the other two. We believe that the main reason for it is the fact that $1 / m_{b}^{3}$ and higher spectator corrections to both $B_{s}$ and $B_{d}$ inclusive widths are given by the same type of diagrams. In other words, apart from the different charm-quark mass dependence (which comes at the level of $m_{c}^{2} / m_{b}^{2}$ corrections to $1 / m_{b}^{3}$ effects), both widths of $B_{s}$ and $B_{d}$ are affected by the weak scattering contributions in the same way. Thus, besides possible $S U(3)$-breaking effects in the matrix 


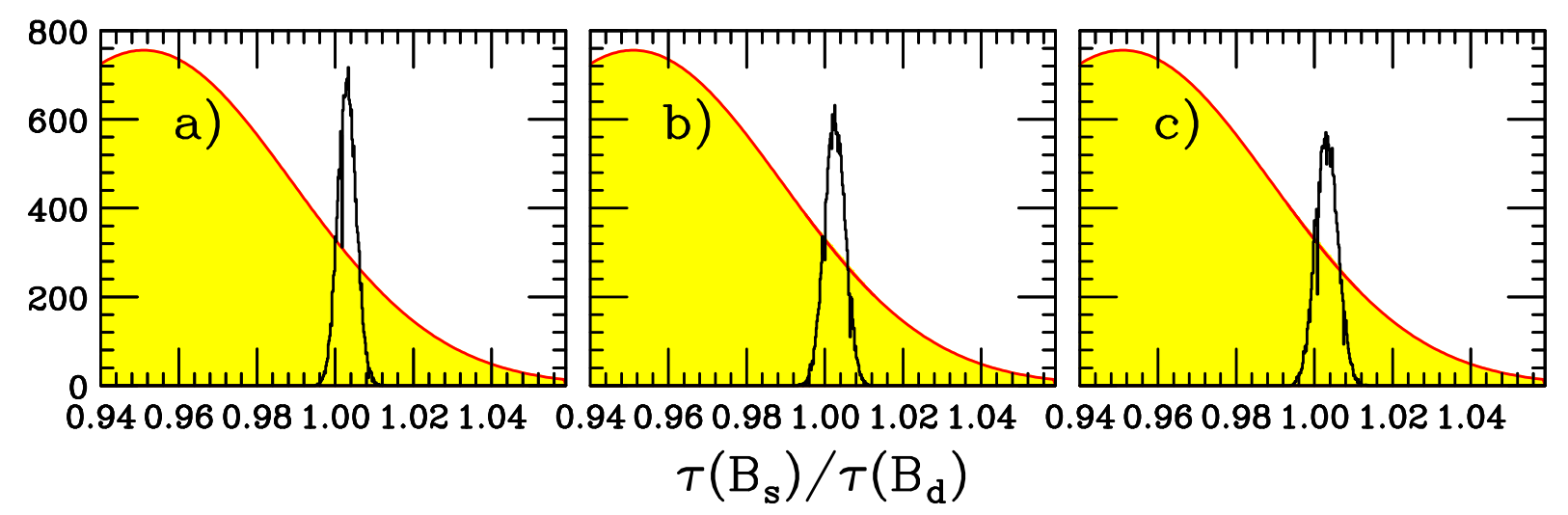

Figure 8: Same as Fig. 6 for $\tau\left(B_{s}\right) / \tau\left(B_{d}\right)$.

elements of the 4-fermion operators, the contributions of spectator interactions to the numerator and denominator of the ratio of $B_{s}$ and $B_{d}$ widths cancel each other to a large degree. This effect persists for the class of $1 / m_{b}$ corrections to spectator effects discussed here as well.

\section{Conclusions}

We computed subleading $1 / m_{b}$ and $1 / m_{b}^{2}$ corrections to the spectator effects driving the difference in the lifetimes of heavy mesons and baryons. Thanks to the same $16 \pi^{2}$ phase-space enhancement as $1 / m_{b}^{3}$-suppressed spectator effects, these corrections constitute the most important set of $1 / m_{b}^{4}$ suppressed corrections.

We showed that a set of $1 / m_{b}$-corrections to spectators effects can be parametrized by eight new parameters $\beta_{i}$ and $\widetilde{\beta}_{i}(i=1, \ldots, 4)$ for heavy mesons and eight new parameters $\beta_{i}^{\Lambda}$ and $\widetilde{\beta}_{i}^{\Lambda}$ for heavy baryons. Although model-independent values of these parameters will not be known until dedicated lattice simulations are performed, we present an estimate of these parameters based on quark model arguments. Compared to [7], we kept all matrix elements, including those vanishing in the factorization approximation. This is important, as the Wilson coefficients of those operators are larger than the ones multiplying the operators whose matrix elements survive in the $N_{c} \rightarrow \infty$ limit. We also perform studies of convergence of this expansion by computing a set of $1 / \mathrm{m}_{b^{-}}^{2}$ corrections to spectator effects and estimating their size in factorization. The expansion appears to be well-convergent.

The main result of this paper are Figs. 6, 7, and 8, which represent the effects of subleading spectator effects on the ratios of lifetimes of heavy mesons and baryons. We see that subleading corrections to spectator effects affect the ratio of heavy meson lifetimes only modestly, at the level of a fraction of a percent. On the other hand, the effect on the $\Lambda_{b}-B_{d}$ lifetime ratio is quite substantial, at the level of $-3 \%$. This can be explained by the partial cancellation of WS and PI effects in $\Lambda_{b}$ baryon and constructive interference of $1 / m_{b}$ corrections to the spectator effects.

There is no theoretically-consistent way to translate the histograms of Figs. 6, 7, and 8 into numerical predictions for the lifetime ratios. As a useful estimate it is possible to fit the histograms to Gaussian distributions and extract theoretical predictions for the mean values and deviations 
of the ratios of lifetimes. Predictions obtained this way should be treated with care, as it is not expected that the theoretical predictions are distributed according to the Gaussian distribution. This being said, we proceed by fitting the distributions to Gaussian and, correcting for the scale uncertainty [varied in the interval $\left(m_{b} / 2,2 m_{b}\right)$ ] by inflating the error bars, extract the ratios $\tau\left(B_{u}\right) / \tau\left(B_{d}\right)=1.06 \pm 0.02, \tau\left(B_{s}\right) / \tau\left(B_{d}\right)=1.00 \pm 0.01$, and $\tau\left(\Lambda_{b}\right) / \tau\left(B_{d}\right)=0.86 \pm 0.05$. In these equations the mean values are taken at the scale $\mu=m_{b}$. This brings the experimental and theoretical ratios of baryon and meson lifetimes into agreement.

\section{Acknowledgments}

We would like to thank N. Uraltsev and M. Voloshin for helpful discussions and A. Lenz for pointing out a numerical mistake in the first version of the manuscript. A.A.P. thanks the Fermilab Theory Group for hospitality where part of this work was completed. This work was supported in part by the U.S. National Science Foundation under Grant PHY-0244853, and by the U.S. Department of Energy under Contract DE-FG02-96ER41005.

\section{Appendix A. Four-quark operators}

This paper uses the basis of $1 / m_{b}^{3}$ operators consistent with Ref. [3], but different from Refs. [4,6], given the relative simplicity of the higher-order operators in our basis. In the above calculations we used the following notation for local four-quark operators:

$$
\begin{aligned}
& O_{1}^{q}=\bar{b}_{i} \gamma^{\mu}\left(1-\gamma_{5}\right) b_{i} \bar{q}_{j} \gamma_{\mu}\left(1-\gamma_{5}\right) q_{j}, \quad O_{2}^{q}=\bar{b}_{i} \gamma^{\mu} \gamma_{5} b_{i} \bar{q}_{j} \gamma_{\mu}\left(1-\gamma_{5}\right) q_{j}, \\
& O_{3}^{q}=\bar{b}_{i} \gamma^{\mu}\left(1-\gamma_{5}\right) b_{i} \bar{q}_{j} \gamma_{\mu} \gamma_{5} q_{j},
\end{aligned}
$$

as well as $\widetilde{O}_{i}^{q}$ operators that follow from the expressions for $O_{i}^{q}$ by interchanging the color indexes of $b_{i}$ and $q_{j}$ Dirac spinors. Refs. $[4,6]$ use more familiar "color-singlet" and "color-octet" operators

$$
\begin{array}{ll}
Q^{q}=\bar{b}_{L} \gamma_{\mu} q_{L} \bar{q}_{L} \gamma^{\mu} b_{L}, & Q_{S}^{q}=\bar{b}_{R} q_{L} \bar{q}_{L} b_{R}, \\
T^{q}=\bar{b}_{L} \gamma_{\mu} t^{a} q_{L} \bar{q}_{L} \gamma^{\mu} t^{a} b_{L}, & T_{S}^{q}=\bar{b}_{R} t^{a} q_{L} \bar{q}_{L} t^{a} b_{R} .
\end{array}
$$

These operators can be expressed in our basis as

$$
\begin{array}{rlrl}
Q^{q} & =\frac{1}{4} \widetilde{O}_{1}^{q}, & Q_{S}^{q} & =-\frac{1}{8} \widetilde{O}_{1}^{q}-\frac{1}{4} \widetilde{O}_{2}^{q}, \\
T^{q}=-\frac{1}{8 N_{c}} \widetilde{O}_{1}^{q}+\frac{1}{8} O_{1}^{q}, & T_{S}^{q}=\frac{1}{8 N_{c}}\left(\frac{1}{2} \widetilde{O}_{1}^{q}+\widetilde{O}_{2}^{q}\right)-\frac{1}{8}\left(\frac{1}{2} O_{1}^{q}+O_{2}^{q}\right) .
\end{array}
$$

Note that in the predictions for lifetime ratios, large matrix elements of the "color-singlet" operators $Q^{q}$ and $Q_{S}^{q}$ are multiplied by a relatively small combination of Wilson coefficients, while matrix elements of "color-octet" operators come with large Wilson coefficients $[4,14]$. Nevertheless, the interpretation of matrix elements obtained in the vacuum insertion approximation (i.e. 
representing the matrix elements of four-fermion operators as products of two matrix elements of current operators separated by a vacuum state) is relatively clear in this basis. For the dimension- 6 operators this procedure introduces four new scale-dependent parameters $B_{i}(\mu)$ and $\epsilon_{i}(\mu)$,

$$
\begin{aligned}
& \frac{1}{2 m_{B_{q}}}\left\langle B_{q}\left|Q^{q}\right| B_{q}\right\rangle=\frac{f_{B_{q}}^{2} m_{B_{q}}}{8} B_{1}(\mu), \quad \frac{1}{2 m_{B_{q}}}\left\langle B_{q}\left|Q_{S}^{q}\right| B_{q}\right\rangle=\frac{f_{B_{q}}^{2} m_{B_{q}}}{8} B_{2}(\mu), \\
& \frac{1}{2 m_{B_{q}}}\left\langle B_{q}\left|T^{q}\right| B_{q}\right\rangle=\frac{f_{B_{q}}^{2} m_{B_{q}}}{8} \epsilon_{1}(\mu), \quad \frac{1}{2 m_{B_{q}}}\left\langle B_{q}\left|T_{S}^{q}\right| B_{q}\right\rangle=\frac{f_{B_{q}}^{2} m_{B_{q}}}{8} \epsilon_{2}(\mu) .
\end{aligned}
$$

These parameters can be computed using QCD sum rules, quark models, or on the lattice. A compilation of various estimates of these parameters can be found in [17].

\section{Appendix B. Estimate of matrix elements}

We parametrize relevant matrix elements using factorization results as a guiding principle (a similar approach was used in $[4,7,18])$. Of course, these matrix elements would eventually have to be computed model-independently, using lattice QCD or QCD sum rules. In the absence of model-independent results, we obtain numerical predictions for the ratios of lifetimes, by relating matrix elements of the computed operators constituting $1 / m_{b}$ and $1 / m_{b}^{2}$ effects to the matrix elements of the operators of dimension $6\left(O_{1,2}\right)$ using quark model. We then use lattice QCD predictions for the parameters $B_{i}$ and $\epsilon_{i}$ and look at the ratio of the subleading- and leading-order spectator effects. We believe that some errors introduced by our use of the quark model should partially cancel in these ratios. The leading-order meson matrix elements are

$$
\begin{aligned}
& \left\langle B_{q}\left|O_{1}^{q}\right| B_{q}\right\rangle=f_{B_{q}}^{2} m_{B_{q}}^{2}\left(2 \epsilon_{1}+\frac{B_{1}}{N_{c}}\right), \quad\left\langle B_{q}\left|\widetilde{O}_{1}^{q}\right| B_{q}\right\rangle=f_{B_{q}}^{2} m_{B_{q}}^{2} B_{1}, \\
& \left\langle B_{q}\left|O_{2}^{q}\right| B_{q}\right\rangle=-f_{B_{q}}^{2} m_{B_{q}}^{2}\left[\frac{m_{B_{q}}^{2}}{\left(m_{b}+m_{q}\right)^{2}}\left(2 \epsilon_{2}+\frac{B_{2}}{N_{c}}\right)+\frac{1}{2}\left(2 \epsilon_{1}+\frac{B_{1}}{N_{c}}\right)\right], \\
& \left\langle B_{q}\left|\widetilde{O}_{2}^{q}\right| B_{q}\right\rangle=-f_{B_{q}}^{2} m_{B_{q}}^{2}\left[\frac{m_{B_{q}}^{2}}{\left(m_{b}+m_{q}\right)^{2}} B_{2}+\frac{1}{2} B_{1}\right], \\
& \left\langle B_{q}\left|O_{3}^{q}\right| B_{q}\right\rangle=\left\langle B_{q}\left|O_{2}^{q}\right| B_{q}\right\rangle, \quad\left\langle B_{q}\left|\widetilde{O}_{3}^{q}\right| B_{q}\right\rangle=\left\langle B_{q}\left|\widetilde{O}_{2}^{q}\right| B_{q}\right\rangle .
\end{aligned}
$$

The parameters $B_{i}$ and $\epsilon_{i}$ represent matrix elements of "color-singlet" and "color-octet" operators. The above parameters can be computed in QCD sum rules, quark models, or on the lattice. Naively, one expects that in the large- $N_{c}$ limit $B_{i} \sim \mathcal{O}(1), \epsilon_{i} \sim \mathcal{O}\left(1 / N_{c}\right)$.

A parametrization of $1 / m_{b}$-suppressed corrections could, in principle, be quite simple: after scaling out the proper $m_{b}$-dependence each new matrix element brings in a new parameter. This parametrization could be useful for lattice studies of these matrix elements. Here we shall choose a slightly different approach. Using the quark model to guide our parametrization, we relate our matrix elements to the matrix elements of singlet and octet operators using Fierz relations. We 
find

$$
\begin{aligned}
& \left\langle B_{q}\left|R_{0}^{q}\right| B_{q}\right\rangle=-\frac{1}{2} f_{B_{q}}^{2} m_{B_{q}}^{2}\left(2 \widetilde{\beta}_{0}+\frac{\beta_{0}}{N_{c}}\right)\left(\frac{m_{B_{q}}^{2}}{m_{b}^{2}}-1\right), \\
& \left\langle B_{q}\left|\widetilde{R}_{0}^{q}\right| B_{q}\right\rangle=-\frac{1}{2} f_{B_{q}}^{2} m_{B_{q}}^{2} \beta_{0}\left(\frac{m_{B_{q}}^{2}}{m_{b}^{2}}-1\right), \\
& \left\langle B_{q}\left|R_{1}^{q}\right| B_{q}\right\rangle=\frac{1}{2} f_{B_{q}}^{2} m_{B_{q}}^{2}\left(\frac{\beta_{1}}{N_{c}}+2 \widetilde{\beta_{1}}\right)\left(\frac{m_{B_{q}}^{2}}{m_{b}^{2}}-1\right), \\
& \left\langle B_{q}\left|\widetilde{R}_{1}^{q}\right| B_{q}\right\rangle=\frac{1}{2} f_{B_{q}}^{2} m_{B_{q}}^{2} \beta_{1}\left(\frac{m_{B_{q}}^{2}}{m_{b}^{2}}-1\right), \\
& \left\langle B_{q}\left|R_{2}^{q}\right| B_{q}\right\rangle=\frac{1}{12} f_{B_{q}}^{2} m_{B_{q}}^{2}\left(\frac{\beta_{2}}{N_{c}}+2 \widetilde{\beta_{2}}\right)\left(\frac{m_{B_{q}}^{2}}{m_{b}^{2}}-1\right), \\
& \left\langle B_{q}\left|\widetilde{R}_{2}^{q}\right| B_{q}\right\rangle=\frac{1}{12} f_{B_{q}}^{2} m_{B_{q}}^{2} \beta_{2}\left(\frac{m_{B_{q}}^{2}}{m_{b}^{2}}-1\right), \\
& \left\langle B_{q}\left|R_{3}^{q}\right| B_{q}\right\rangle=\frac{1}{12} f_{B_{q}}^{2} m_{B_{q}}^{2}\left(\frac{\beta_{3}}{N_{c}}+2 \widetilde{\beta_{3}}\right)\left(\frac{m_{B_{q}}^{2}}{m_{b}^{2}}-1\right), \\
& \left\langle B_{q}\left|\widetilde{R}_{3}^{q}\right| B_{q}\right\rangle=\frac{1}{12} f_{B_{q}}^{2} m_{B_{q}}^{2} \beta_{3}\left(\frac{m_{B_{q}}^{2}}{m_{b}^{2}}-1\right)
\end{aligned}
$$

In the large $N_{c}$-limit $\beta_{i} \sim \mathcal{O}(1)$ and $\widetilde{\beta}_{i} \sim \mathcal{O}\left(1 / N_{c}\right)$. We can use the quark model to relate $\beta_{0}=\beta_{0}^{\prime}\left(B_{2}+B_{1} / 2\right), \beta_{1}=\beta_{1}^{\prime} B_{1}, \beta_{2,3}=\beta_{2,3}^{\prime}\left[\left(B_{1}-4 B_{2}\right)\left(1+m_{b}^{2} / m_{B_{q}}^{2}\right)+\left(B_{1}+2 B_{2}\right)\right]$. The same relations hold for $\widetilde{\beta}_{i}$ with $B_{i} \rightarrow \epsilon_{i}$. Here $\beta_{i}^{\prime}$ are used to parameterize the ratios of the matrix elements of the four fermion operators and $1 / m_{b}$-suppressed operators. In our numerical studies we took the parameters $B_{i}, \epsilon_{i}$ from the lattice calculations of matrix elements of dimension- 6 operators [6] and varied $\beta_{i}^{\prime}$ around its quark-model value of unity by $\pm 30 \%$.

A similar logic can be used to estimate $1 / m_{b}^{2}$-suppressed matrix elements. We simplify the result by using the factorization approximation, i.e. neglecting all the matrix elements of octet operators, which should suffice for our purposes of studying the convergence of the series. We obtain

$$
\begin{aligned}
\left\langle B_{q}\left|W_{1}^{q}\right| B_{q}\right\rangle & =\frac{f_{B_{q}}^{2} m_{B_{q}}^{2}}{4 N_{c}}\left(\frac{m_{B_{q}}^{2}}{m_{b}^{2}}-1\right)^{2}, \\
\left\langle B_{q}\left|\widetilde{W}_{1}^{q}\right| B_{q}\right\rangle & =\frac{f_{B_{q}}^{2} m_{B_{q}}^{2}}{4}\left(\frac{m_{B_{q}}^{2}}{m_{b}^{2}}-1\right)^{2}, \\
\left\langle B_{q}\left|W_{2}^{q}\right| B_{q}\right\rangle & =-\frac{3 f_{B_{q}}^{2} m_{B_{q}}^{2}}{8 N_{c}}\left(\frac{m_{B_{q}}^{2}}{m_{b}^{2}}-1\right)^{2},
\end{aligned}
$$




$$
\begin{aligned}
& \left\langle B_{q}\left|\widetilde{W}_{2}^{q}\right| B_{q}\right\rangle=-\frac{3 f_{B_{q}}^{2} m_{B_{q}}^{2}}{8}\left(\frac{m_{B_{q}}^{2}}{m_{b}^{2}}-1\right)^{2}, \\
& \left\langle B_{q}\left|W_{3}^{q}\right| B_{q}\right\rangle=-\frac{f_{B_{q}}^{2} m_{b}^{2}}{8 N_{c}}\left(\frac{m_{B_{q}}^{2}}{m_{b}^{2}}-1\right)^{2}, \\
& \left\langle B_{q}\left|\widetilde{W}_{3}^{q}\right| B_{q}\right\rangle=-\frac{f_{B_{q}}^{2} m_{b}^{2}}{8}\left(\frac{m_{B_{q}}^{2}}{m_{b}^{2}}-1\right)^{2}, \\
& \left\langle B_{q}\left|W_{4}^{q}\right| B_{q}\right\rangle=\left\langle B_{q}\left|W_{3}^{q}\right| B_{q}\right\rangle,\left\langle B_{q}\left|\widetilde{W}_{4}^{q}\right| B_{q}\right\rangle=\left\langle B_{q}\left|\widetilde{W}_{3}^{q}\right| B_{q}\right\rangle .
\end{aligned}
$$

In a similar way, we used the quark-diquark valence-quark model to guide our parametrizations of baryon matrix elements. The leading order matrix elements are $[4,7]$

$$
\begin{aligned}
\left\langle\Lambda_{b}\left|O_{1}^{q}\right| \Lambda_{b}\right\rangle & =-\widetilde{B}\left\langle\Lambda_{b}\left|\widetilde{O}_{1}^{q}\right| \Lambda_{b}\right\rangle=\frac{\widetilde{B}}{6} f_{B_{q}}^{2} m_{B_{q}} m_{\Lambda_{b}} r, \\
\left\langle\Lambda_{b}\left|O_{2}^{q}\right| \Lambda_{b}\right\rangle & =-\widetilde{B}\left\langle\Lambda_{b}\left|\widetilde{O}_{2}^{q}\right| \Lambda_{b}\right\rangle=\frac{\widetilde{B}}{6} f_{B_{q}}^{2} m_{B_{q}} m_{\Lambda_{b}} \delta \\
\left\langle\Lambda_{b}\left|O_{3}^{q}\right| \Lambda_{b}\right\rangle & =-\widetilde{B}\left\langle\Lambda_{b}\left|\widetilde{O}_{3}^{q}\right| \Lambda_{b}\right\rangle=\left\langle\Lambda_{b}\left|O_{2}^{q}\right| \Lambda_{b}\right\rangle .
\end{aligned}
$$

where $r=\left|\psi_{b q}^{\Lambda_{b}}(0)\right|^{2} /\left|\psi_{b \bar{q}}^{B_{q}}(0)\right|^{2}$ is the ratio of the wave functions at the origin of the $\Lambda_{b}$ and $B_{q}$ mesons, and $\widetilde{B}=1$ in the valence-quark model. Estimates of $r$ vary from 0.1 to 1.8 and can potentially be larger [4]. Note that $\delta=\mathcal{O}\left(1 / m_{b}\right)$, which follows from the heavy-quark spin symmetry. We use the same principles to parametrize the $1 / m_{b}$-suppressed contributions,

$$
\begin{aligned}
& \left\langle\Lambda_{b}\left|R_{0}^{q}\right| \Lambda_{b}\right\rangle=-\frac{\beta_{0}^{\Lambda}}{\widetilde{\beta}_{0}^{\Lambda}}\left\langle\Lambda_{b}\left|\widetilde{R}_{0}^{q}\right| \Lambda_{b}\right\rangle=-\frac{\beta_{0}^{\Lambda}}{24} f_{B_{q}}^{2} m_{B_{q}} m_{\Lambda_{b}}\left(\frac{m_{\Lambda_{b}}^{2}}{m_{b}^{2}}-1\right), \\
& \left\langle\Lambda_{b}\left|R_{1}^{q}\right| \Lambda_{b}\right\rangle=-\frac{\beta_{1}^{\Lambda}}{\widetilde{\beta}_{1}^{\Lambda}}\left\langle\Lambda_{b}\left|\widetilde{R}_{1}^{q}\right| \Lambda_{b}\right\rangle=-\frac{\beta_{1}^{\Lambda}}{24} f_{B_{q}}^{2} m_{B_{q}} m_{\Lambda_{b}}\left(\frac{m_{\Lambda_{b}}^{2}}{m_{b}^{2}}-1\right), \\
& \left\langle\Lambda_{b}\left|R_{2}^{q}\right| \Lambda_{b}\right\rangle=-\frac{\beta_{2}^{\Lambda}}{\widetilde{\beta}_{2}^{\Lambda}}\left\langle\Lambda_{b}\left|\widetilde{R}_{2}^{q}\right| \Lambda_{b}\right\rangle=-\frac{\beta_{2}^{\Lambda}}{48} f_{B_{q}}^{2} m_{B_{q}} m_{\Lambda_{b}}\left(\frac{m_{\Lambda_{b}}^{2}}{m_{b}^{2}}-1\right), \\
& \left\langle\Lambda_{b}\left|R_{3}^{q}\right| \Lambda_{b}\right\rangle=-\frac{\beta_{3}^{\Lambda}}{\widetilde{\beta}_{3}^{\Lambda}}\left\langle\Lambda_{b}\left|\widetilde{R}_{3}^{q}\right| \Lambda_{b}\right\rangle=\frac{\beta_{3}^{\Lambda}}{48} f_{B_{q}}^{2} m_{B_{q}} m_{\Lambda_{b}}\left(\frac{m_{\Lambda_{b}}^{2}}{m_{b}^{2}}-1\right) .
\end{aligned}
$$

Note that the matrix elements contain the parameters $\beta_{0}^{\Lambda}$ and $\widetilde{\beta}_{0}^{\Lambda}$, which are of the order of $1 / m_{b}$. Thus, these matrix elements only contribute at the order $1 / m_{b}^{2}$ corrections to spectator effects (see below). In the valence-quark approximation these parameters are proportional to $\delta$. Analogously to the meson case, the quark model relates $\beta_{0}^{\Lambda}=\beta_{0}^{\prime \Lambda} \widetilde{B} \delta, \widetilde{\beta}_{0}^{\Lambda}=\widetilde{\beta}_{0}^{\Lambda^{\prime}} \delta, \beta_{1}^{\Lambda}=\beta_{1}^{\Lambda \Lambda} \widetilde{B} r, \widetilde{\beta}_{1}^{\Lambda}=\widetilde{\beta}_{1}^{\Lambda^{\prime}} r$, $\beta_{2,3}^{\Lambda}=\beta_{2,3}^{\Lambda^{\prime}} \widetilde{B}\left[(r+4 \delta / 3)\left(1+m_{b}^{2} / m_{\Lambda_{b}}^{2}\right)-2 \delta / 3\right], \widetilde{\beta}_{2,3}^{\Lambda}=\widetilde{\beta}_{2,3}^{\Lambda^{\prime}}\left[(r+4 \delta / 3)\left(1+m_{b}^{2} / m_{\Lambda_{b}}^{2}\right)-2 \delta / 3\right]$. We use the valence-quark approximation for $1 / m_{b}^{2}$ corrections. We obtain

$$
\left\langle\Lambda_{b}\left|W_{1}^{q}\right| \Lambda_{b}\right\rangle=-\left\langle\Lambda_{b}\left|\widetilde{W}_{1}^{q}\right| \Lambda_{b}\right\rangle=\frac{f_{B_{q}}^{2} m_{B_{q}} m_{\Lambda_{b}}}{96}\left(\frac{m_{\Lambda_{b}}^{2}}{m_{b}^{2}}-1\right)^{2},
$$




$$
\begin{aligned}
& \left\langle\Lambda_{b}\left|W_{2}^{q}\right| \Lambda_{b}\right\rangle=-\left\langle\Lambda_{b}\left|\widetilde{W}_{2}^{q}\right| \Lambda_{b}\right\rangle=0 \\
& \left\langle\Lambda_{b}\left|W_{3}^{q}\right| \Lambda_{b}\right\rangle=-\left\langle\Lambda_{b}\left|\widetilde{W}_{3}^{q}\right| \Lambda_{b}\right\rangle=\frac{f_{B_{q}}^{2} m_{B_{q}} m_{\Lambda_{b}}}{192}\left(\frac{m_{\Lambda_{b}}^{2}}{m_{b}^{2}}-1\right)^{2}\left(1+\frac{m_{b}^{2}}{m_{\Lambda_{b}}^{2}}\right), \\
& \left\langle\Lambda_{b}\left|W_{4}^{q}\right| \Lambda_{b}\right\rangle=-\left\langle\Lambda_{b}\left|\widetilde{W}_{4}^{q}\right| \Lambda_{b}\right\rangle=-\left\langle\Lambda_{b}\left|W_{3}^{q}\right| \Lambda_{b}\right\rangle
\end{aligned}
$$

where we set all the terms of order $\mathcal{O}\left(1 / m_{b}^{3}\right)$ in the matrix elements to zero.

\section{References}

[1] S. Eidelman et al., Phys. Lett. B592, 1 (2004); E. Barberio, presented at the Workshop on the CKM Unitary Triangle, http://ckm-workshop.web.cern.ch; M. Battaglia et al., arXiv:hep$\mathrm{ph} / 0304132$.

[2] J. Rademacker [On behalf of the CDF Collaboration], arXiv:hep-ex/0406021. See also: LEP B Lifetime Working Group, http://lepbosc.web.cern.ch/LEPBOSC/lifetimes/lepblife.html.

[3] I. I. Bigi et al., arXiv:hep-ph/9401298; M. B. Voloshin, arXiv:hep-ph/0004257.

[4] M. Neubert and C. T. Sachrajda, Nucl. Phys. B 483, 339 (1997).

[5] J. L. Rosner, Phys. Lett. B 379, 267 (1996).

[6] M. Ciuchini, E. Franco, V. Lubicz, and F. Mescia, Nucl. Phys. B 625, 211 (2002); E. Franco, V. Lubicz, F. Mescia, and C. Tarantino, ibid. 633, 212 (2002).

[7] F. Gabbiani, A. I. Onishchenko, and A. A. Petrov, Phys. Rev. D 68, 114006 (2003).

[8] I. I. Y. Bigi, M. A. Shifman, N. G. Uraltsev and A. I. Vainshtein, Phys. Rev. D 50, 2234 (1994).

[9] C. Tarantino, arXiv:hep-ph/0310241.

[10] I. I. Bigi, M. Shifman, N. Uraltsev, and A. Vainshtein, Phys. Rev. Lett. 71, 496 (1993).

[11] B. Guberina, S. Nussinov, R. D. Peccei, and R. Ruckl, Phys. Lett. B 89, 111 (1979).

[12] N. Bilic, B. Guberina, and J. Trampetic, Nucl. Phys. B 248, 261 (1984).

[13] B. Guberina, R. Ruckl, and J. Trampetic, Z. Phys. C 33, 297 (1986).

[14] M. A. Shifman and M. B. Voloshin, Sov. J. Nucl. Phys. 41, 120 (1985) [Yad. Fiz. 41, 187 (1985)].

[15] M. Beneke, G. Buchalla, C. Greub, A. Lenz, and U. Nierste, Nucl. Phys. B 639, 389 (2002).

[16] B. Guberina, B. Melic, and H. Stefancic, Phys. Lett. B 484, 43 (2000). 
[17] J. Chay, A. F. Falk, M. E. Luke, and A. A. Petrov, Phys. Rev. D 61, 034020 (2000); see also [1] and M. Di Pierro and C. T. Sachrajda [UKQCD Collaboration], Nucl. Phys. B 534, 373 (1998); M. Di Pierro, C. T. Sachrajda, and C. Michael [UKQCD collaboration], Phys. Lett. B 468, 143 (1999); P. Colangelo and F. De Fazio, ibid. 387, 371 (1996); M. S. Baek, J. Lee, C. Liu, and H. S. Song, Phys. Rev. D 57, 4091 (1998); J. G. Korner, A. I. Onishchenko, A. A. Petrov, and A. A. Pivovarov, Phys. Rev. Lett. 91, 192002 (2003).

[18] M. Beneke, G. Buchalla, and I. Dunietz, Phys. Rev. D 54, 4419 (1996). M. Beneke, G. Buchalla, A. Lenz, and U. Nierste, Phys. Lett. B 576, 173 (2003). 